\title{
HIGH-RESOLUTION RADIO CONTINUUM MEASUREMENTS OF THE NUCLEAR DISKS OF Arp 220
}

\author{
L. Barcos-Muñoz ${ }^{1}$, A. K. Leroy ${ }^{2}$, A. S. Evans ${ }^{1,2}$, G. C. Privon ${ }^{1}$, L. Armus ${ }^{3}$, J. Condon ${ }^{2}$, J. M. Mazzarella ${ }^{4}$, \\ D. S. Meier ${ }^{5,6}$, E. Momjian ${ }^{6}$, E. J. Murphy ${ }^{4}$, J. OtT ${ }^{6}$, A. ReichardT ${ }^{2,12}$, K. SAKAmoto $^{7}$, D. B. SAnders ${ }^{8}$, \\ E. Schinnerer ${ }^{9}$, S. Stierwalt ${ }^{1}$, J. A. Surace ${ }^{10}$, T. A. Thompson ${ }^{11}$, And F. Walter ${ }^{9}$ \\ ${ }^{1}$ Department of Astronomy, University of Virginia, 530 McCormick Road, Charlottesville, VA 22904, USA; ldb7et@ virginia.edu \\ ${ }^{2}$ National Radio Astronomy Observatory, 520 Edgemont Road, Charlottesville, VA 22904, USA \\ ${ }^{3}$ Spitzer Science Center, California Institute of Technology, MC 220-6, 1200 East California Boulevard, Pasadena, CA 91125, USA \\ ${ }^{4}$ Infrared Processing and Analysis Center, MS 100-22, California Institute of Technology, Pasadena, CA 91125, USA \\ ${ }^{5}$ New Mexico Institute of Mining and Technology, 801 Leroy Place, Socorro, NM 87801, USA \\ ${ }^{6}$ National Radio Astronomy Observatory, P.O. Box O, 1003 Lopezville Road, Socorro, NM 87801, USA \\ ${ }^{7}$ Academia Sinica, Institute of Astronomy and Astrophysics, P.O. Box 23-141, Taipei 10617, Taiwan \\ 8 Institute for Astronomy, University of Hawaii, 2680 Woodlawn Drive, Honolulu, HI 96816, USA \\ ${ }^{9}$ Max-Planck-Institut für Astronomie, Königstuhl 17, D-69117 Heidelberg, Germany \\ ${ }^{10}$ Spitzer Science Center, MS 314-6, California Institute of Technology, Pasadena, CA 91125, USA \\ ${ }^{11}$ Department of Astronomy and Center for Cosmology \& Astro-Particle Physics, The Ohio State University, Columbus, OH 43210, USA \\ Received 2014 May 1; accepted 2014 November 3; published 2015 January 12
}

\begin{abstract}
We present new Karl G. Jansky Very Large Array radio continuum images of the nuclei of Arp 220, the nearest ultraluminous infrared galaxy. These new images have both the angular resolution to study the detailed morphologies of the two nuclei that power the galaxy merger and sensitivity to a wide range of spatial scales. At $33 \mathrm{GHz}$, we achieve a resolution of $0^{\prime} .081 \times 0^{\prime \prime} 063(29.9 \times 23.3 \mathrm{pc})$ and resolve the radio emission surrounding both nuclei. We conclude from the decomposition of the radio spectral energy distribution that a majority of the $33 \mathrm{GHz}$ emission is synchrotron radiation. The spatial distributions of radio emission in both nuclei are well described by exponential profiles. These have deconvolved half-light radii $\left(R_{50 d}\right)$ of 51 and $35 \mathrm{pc}$ for the eastern and western nuclei, respectively, and they match the number density profile of radio supernovae observed with very long baseline interferometry. This similarity might be due to the fast cooling of cosmic rays electrons caused by the presence of a strong $(\sim \mathrm{mG})$ magnetic field in this system. We estimate extremely high molecular gas surface densities of $2.2_{-1.0}^{+2.1} \times 10^{5}$ (east) and $4.5_{-1.9}^{+4.5} \times 10^{5}$ (west) $M_{\odot} \mathrm{pc}^{-2}$, corresponding to total hydrogen column densities of $N_{\mathrm{H}}=2.7_{-1.2}^{+2.7} \times 10^{25}$ (east) and $5.6_{-2.4}^{+5.5} \times 10^{25} \mathrm{~cm}^{-2}$ (west). The implied gas volume densities are similarly high, $n_{H_{2}} \sim 3.8_{-1.6}^{+3.8} \times 10^{4}$ (east) and $\sim 11_{-4.5}^{+12} \times 10^{4} \mathrm{~cm}^{-3}$ (west). We also estimate very high luminosity surface densities of $\Sigma_{\mathrm{IR}} \sim 4.2_{-0.7}^{+1.6} \times 10^{13}$ (east) and $\Sigma_{\mathrm{IR}} \sim 9.7_{-2.4}^{+3.7} \times 10^{13}$ (west) $L_{\odot} \mathrm{kpc}^{-2}$, and star formation rate surface densities of $\Sigma_{\mathrm{SFR}} \sim 10^{3.7 \pm 0.1}$ (east) and $\Sigma_{\mathrm{SFR}} \sim 10^{4.1 \pm 0.1}$ (west) $M_{\odot} \mathrm{yr}^{-1} \mathrm{kpc}^{-2}$. These values, especially for the western nucleus are, to our knowledge, the highest luminosity surface densities and star formation rate surface densities measured for any star-forming system. Despite these high values, the nuclei appear to lie below the dusty Eddington limit in which radiation pressure is balanced only by self-gravity. The small measured sizes also imply that at wavelengths shorter than $\lambda=1 \mathrm{~mm}$, dust absorption effects must play an important role in the observed light distribution while below $5 \mathrm{GHz}$ free-free absorption contributes substantial opacity. According to these calculations, the nuclei of Arp 220 are only transparent in the frequency range $\sim 5-350 \mathrm{GHz}$. Our results offer no clear evidence that an active galactic nucleus dominates the emission from either nucleus at $33 \mathrm{GHz}$.
\end{abstract}

Key words: galaxies: active - galaxies: individual (Arp 220) - galaxies: interactions - galaxies: starburst radio continuum: galaxies

\section{INTRODUCTION}

Starbursts induced by major mergers are among the most extreme environments in the universe. Despite their prodigious luminosities, local merger-driven starbursts are very compact, with most of their large gas reservoirs concentrated in dusty regions a few hundred parsecs, or less, in size (e.g., Downes \& Solomon 1998). Measuring the compactness of these starbursts is critical to understanding these galaxies (e.g., Soifer et al. 1999, 2000; Sakamoto et al. 2008; Díaz-Santos et al. 2013). Robust size measurements allow us to translate luminosities into key physical quantities such as gas column density, optical depth, volumetric gas density, and star formation rate (SFR) and luminosity surface densities. Although their luminosity renders them visible out to great distances, the

\footnotetext{
12 NRAO NSF-sponsored REU student.
}

present-day rarity of major mergers means that even the nearest ultra-luminous infrared galaxies (ULIRGs: defined as having $L_{\mathrm{IR}}[8-1000 \mu \mathrm{m}] \geqslant 10^{12} L_{\odot}$ ) are relatively distant (>70 Mpc). Thus, measuring the true extent of their active regions requires high angular resolution. The extraordinary extinctions present in these systems at both long (from free-free absorption) and short (from dust opacity) wavelengths complicate the interpretation of measurements at both wavelengths, compounding the difficulty of measuring sizes for such systems.

Given the above considerations, radio observations at centimeter wavelengths may be the best tool to study the deeply embedded, compact structures at the heart of such systems (e.g., Norris 1988; Condon et al. 1991). Radio interferometers can achieve very high angular resolution and radio waves with $v \gtrsim 5 \mathrm{GHz}$ can penetrate large columns of dust and are largely unaffected by free-free absorption. The recent upgrades to the Karl G. Jansky Very Large Array (VLA) make it particularly 
well suited for such studies. In this paper, we make use of these new VLA capabilities to achieve the best measurement to date of the structure of the nuclear region of the nearest ULIRG, Arp 220.

At a luminosity distance of 77.2 Mpc, and an infrared (IR) luminosity of $L_{\mathrm{IR}}[8-1000 \mu \mathrm{m}]=1.44 \times 10^{12} L_{\odot},{ }^{13} \operatorname{Arp} 220$ is the nearest ULIRG. CO and near-IR observations indicate that Arp 220 is a gas-rich merger with dynamical masses of $\sim 10^{9} M_{\odot}$ within $\sim 100$ pc of each nucleus (Downes \& Solomon 1998; Sakamoto et al. 1999, 2008; Genzel et al. 2001; Engel et al. 2011). Arp 220 is obscured at optical through mid-IR wavelengths (Scoville et al. 1998; Soifer et al. 1999; Haas et al. 2001; Spoon et al. 2007; Armus et al. 2007), obstructing the direct view of the nuclear energy sources at these wavelengths. Observations in the frequency range where Arp 220 is optically thin have been able to resolve the system into two compact nuclear disks (Norris 1988; Condon et al. 1991; Downes \& Solomon 1998; Sakamoto et al. 2008) and find disk sizes of $\sim 0^{\prime \prime} .2$. However, in each case the measured sizes remain comparable to the size of the beam. Very long baseline interferometry (VLBI) observations at centimeter wavelengths by Smith et al. (1998), Lonsdale et al. (2006), and Parra et al. (2007) provide a higher resolution view, recovering a compact distribution of point-like sources that are proposed to be a combination of radio supernovae (RSNe) and supernova remnants (SNRs). However, these observations resolve out most of the emission from the disks. From the above, it is already clear that the disks are very compact, implying extraordinary volume densities and surface densities. The next step is to observe the disks in the optically thin frequency range with resolution high enough to clearly resolve them, and sensitivity to recover the full extent of the emission of the system at that frequency range.

In this paper, we measure the structure of the Arp 220 nuclei with sensitive, high angular resolution images obtained at $6 \mathrm{GHz}$ and $33 \mathrm{GHz}$ observed with the VLA. Based on the integrated spectral energy distribution (SED) model of Arp 220 (see Figure 10(b) in Anantharamaiah et al. 2000), the total continuum flux density at $33 \mathrm{GHz}$ is a mixture of thermal and nonthermal emission with a $\sim 1: 2$ ratio, while at $6 \mathrm{GHz}$ this ratio is about 1:5. Observing at these two frequencies then helps us diagnose the dominant emission mechanism at radio wavelengths in Arp 220. We first report our observations, describe the calculations used to assess the disk structure, and then discuss the implications of our measurements. Throughout this paper, we adopt $\mathrm{H}_{0}=73 \mathrm{~km} \mathrm{~s}^{-1} \mathrm{Mpc}^{-1}, \Omega_{\text {vacuum }}=0.73$, $\Omega_{\text {matter }}=0.27$, and $v_{\text {optical }}=5555 \mathrm{~km} \mathrm{~s}^{-1}$ (after correction to the cosmic microwave background frame), such that $1^{\prime \prime}$ on the sky plane subtends 369 pc at the distance of Arp 220.

\section{OBSERVATIONS}

We observed Arp 220 using the VLA $C$ (4-8 GHz) and $K a$ band $(26.5-40 \mathrm{GHz})$ receivers, recording emission in $1 \mathrm{GHz}$ wide windows centered at $\sim 4.7,7.2,29$, and $36 \mathrm{GHz}$. We used all four VLA configurations with a total integration time ratio of 1:1:2:4 between D (lowest resolution), C, B, and A. The total onsource integration time was 40 minutes at $C$ band and 56 minutes at $\mathrm{Ka}$ band. We used 3C 286 as the flux density and bandpass calibrator, and $\mathrm{J} 1513+2338$ and $\mathrm{J} 1539+2744$ as the complex

\footnotetext{
$13 D_{L}$ from NED; $L_{\mathrm{IR}}$ using Table 1 in Sanders \& Mirabel (1996) and IRAS flux densities from Sanders et al. (2003). However, note that the assumption of isotropic emission that leads to this luminosity has some caveats (see Section 4.3.2 and Appendix A).
}

gain calibrators at $C$ and $K a$ bands, respectively. The data were obtained in multiple observing sessions during the period 2010 August 18 to 2011 July 2, with the $C$ and $K a$ band observations carried out in separate sessions. These observations are part of a larger project; the lower resolution ( $\mathrm{C}$ and $\mathrm{D}$ configuration) results are presented in Leroy et al. (2011) and Murphy (2013), and the final results for the complete sample will be reported in L. Barcos-Muñoz et al. (in preparation).

We reduced the data using the Common Astronomy Software Application (CASA; McMullin et al. 2007) package following the standard procedure for VLA data. Radio frequency interference (RFI) contaminating the $C$ band was eliminated using the task flagdata in mode rflag. The RFI at $K a$ band was negligible. After calibration, we combined the data from all configurations, weighting them in proportion to their integration time per visibility (i.e., 10:5:2:1 for D:C:B:A). We then imaged this combined data using the task CLEAN in mode mfs (Sault \& Wieringa 1994), with Briggs weighting setting robust $=0.5$. We combined all the data within each receiver band and cleaned using components with a variable spectral index (nterms=2) to obtain an interpolated image at an intermediate frequency (5.95 GHz for $C$ band and $32.5 \mathrm{GHz}$ for $K a$ band). Even after the initial calibration, we still observed phase and amplitude variations with time. To improve the images further, we iteratively self-calibrated in both phase and amplitude and applied extra flagging during this procedure as needed. The solutions for the amplitude self-calibration were carefully inspected and accepted as long as the time variations in the amplitude gains for each antenna were less than $\sim 20 \%$. We worked mostly with these "combined" images at 5.95 and $32.5 \mathrm{GHz}$, but we also separately imaged the two $1 \mathrm{GHz}$ windows at $C$ band in order to derive a robust internal $C$-band spectral index.

To check our results, we imaged the $33 \mathrm{GHz}$ data separately for each VLA configuration. In this test, we primarily applied iterative phase self-calibration. Amplitude self-calibration was applied (after $\sim 3-5$ iterations of phase self-calibration), but here we only derived normalized solutions that cannot change the observed flux. During this check, we also experimented with weighting the visibilities by the measured rms noise in each data set (using the CASA task statwt). These tests revealed that the combined $\mathrm{A}+\mathrm{B}$ (two most extended) configurations recovered essentially all of the flux in the data, agreeing with the $C$ and $D$ data in both flux and morphology when convolved to matched resolution. The A configuration data alone recovered less flux than the B configuration, consistent with some spatial filtering at this highest resolution. Furthermore, the overall flux recovered agrees with an interpolation of the integrated SED (Anantharamaiah et al. 2000). We proceed using the full combined image with our confidence in the results reinforced by these tests; we verified that our fitting yields consistent results using the combined image and the $\mathrm{A}+\mathrm{B}$ configuration-only image.

The clean restoring beam for the combined images has a FWHM of $0.48 \times 0.35(177 \times 129 \mathrm{pc})$ at position angle (p.a.) of $-40^{\circ}$ at $6 \mathrm{GHz}(C$ band $)$, and $0^{\prime} .081 \times 0.063(30 \times 23 \mathrm{pc})$ at a p.a. of $65^{\circ}$ at $32.5 \mathrm{GHz}$ ( $\mathrm{Ka}$ band). The rms noise measured from signal-free parts of the image is $\sim 14 \mu \mathrm{Jy}^{\text {beam }}{ }^{-1}(C)$ and $\sim 23 \mu \mathrm{Jy}_{\text {beam }}{ }^{-1}(\mathrm{Ka})$, which is within a factor of two of the expected theoretical noise. The final dynamic ranges of the images are $\sim 5.2 \times 10^{3}$ and $\sim 280$, for $C$ and $K a$ band.

When reporting the measured flux densities from the final images, we consider three sources of uncertainty. First, we propagated the beam-to-beam noise (see above) and found its effect to be negligible at both bands. Second, we assessed the 

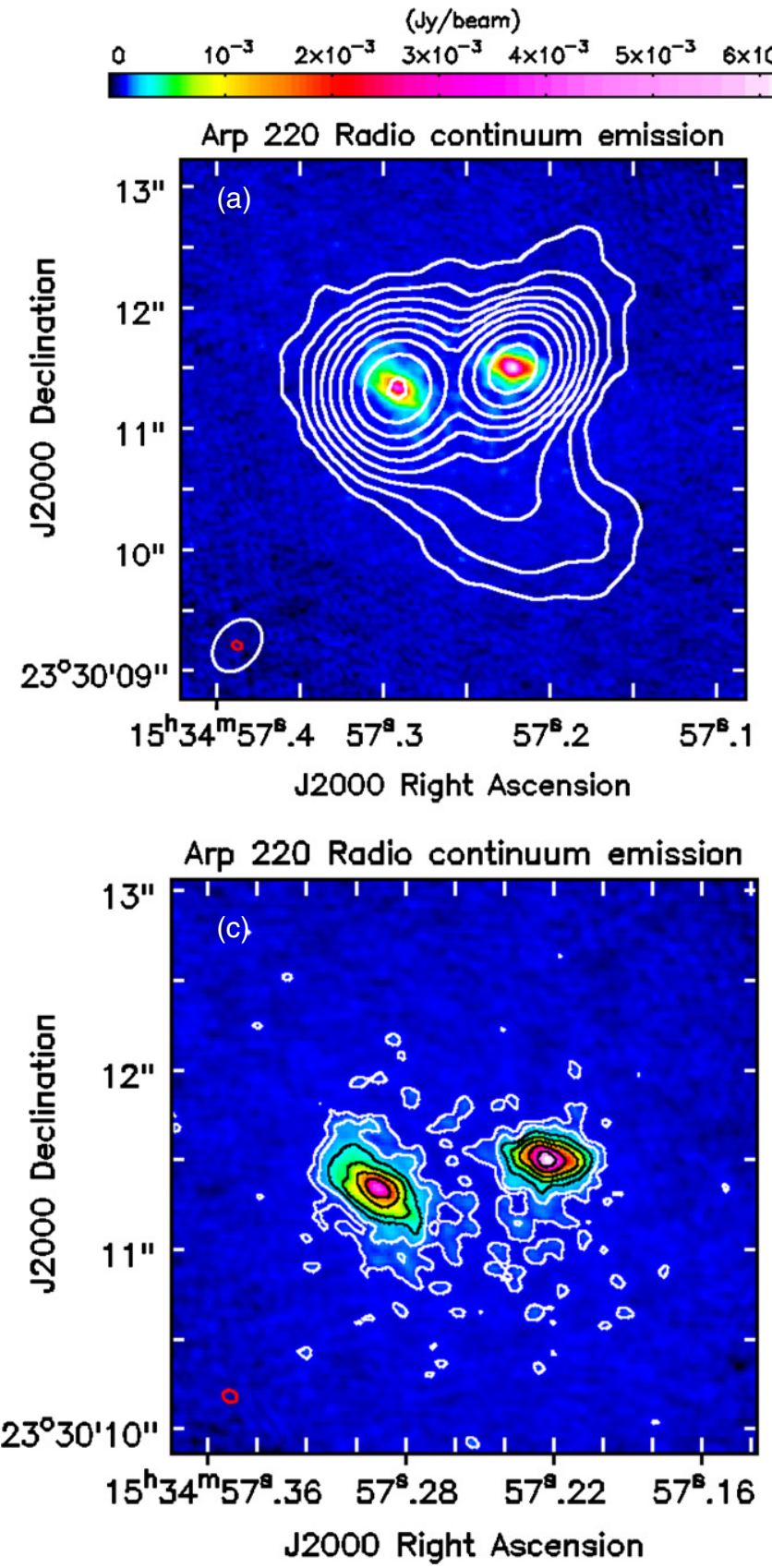

Arp $220 \mathrm{cO}(3-2)$ zeroth moment map

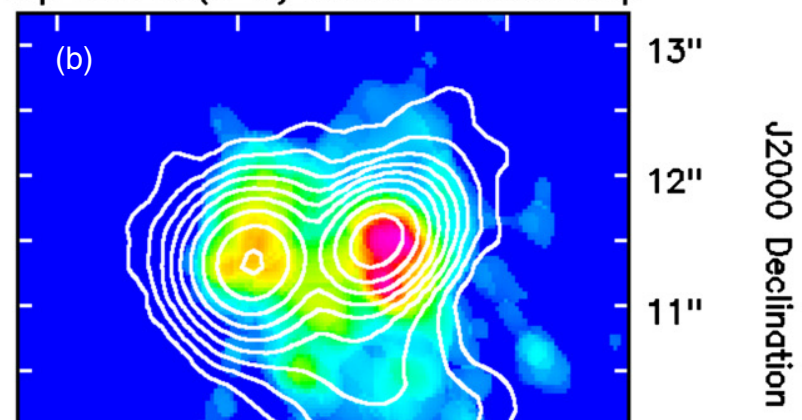

$10^{\prime \prime}$

$23^{\circ} 30^{\prime} 09^{\prime}$

응
8
$\frac{8}{5}$
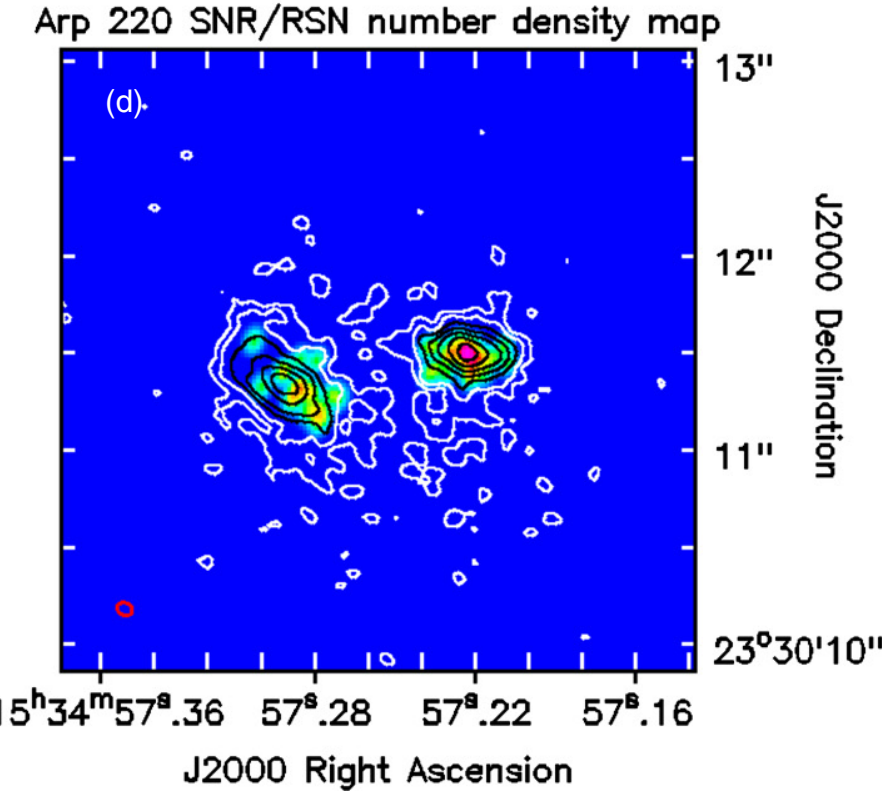

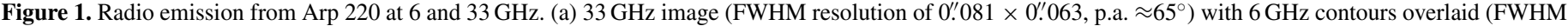

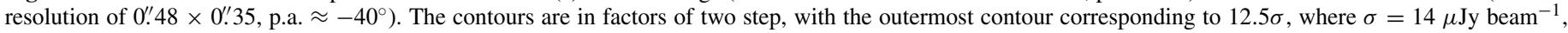

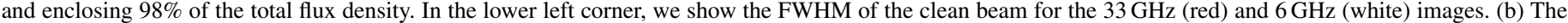

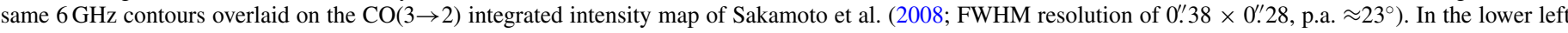

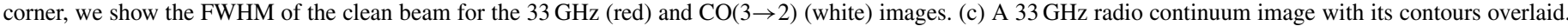

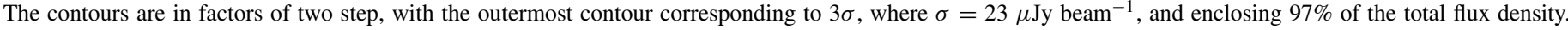

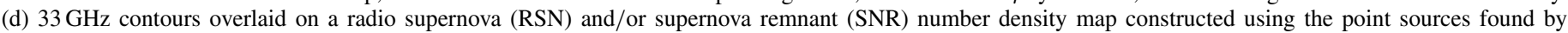

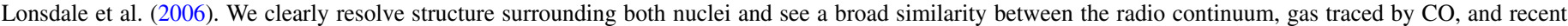
RSNe and/or SNRs.

impact of the curve-of-growth technique used to measure the flux densities (see Section 3) by using the scatter of such a curve. This is also small, but larger for the individual nuclei because they are not perfectly separable. Finally, we estimated the uncertainty in the overall flux density calibration from the day-to-day variation of the flux density of the complex gain calibrator. This scatter (rms) is $\sim 12 \%$ at $K a$ band, making the flux density calibration the dominant source of uncertainty at this band. At $C$ band, the scatter in the curves of growth and the variation in the flux density calibration are comparable, i.e., $\sim 1 \%$. We sum all three uncertainty terms in quadrature and report the combined value in Table 1 . Note that in addition to these uncertainties, the absolute flux scale used at the VLA is estimated to be uncertain by $\approx 2 \%$.

After reducing the data, we compared our $33 \mathrm{GHz}$ image to VLA (Norris 1988; Condon et al. 1991), Submillimeter Array (SMA; Sakamoto et al. 2008), and ALMA archival images (Wilson et al. 2014, and N. Z. Scoville et al. in preparation). We found astrometric discrepancies of order 0'.1 (i.e., 1-2 beams) and traced the origin of these to the adopted position of our 

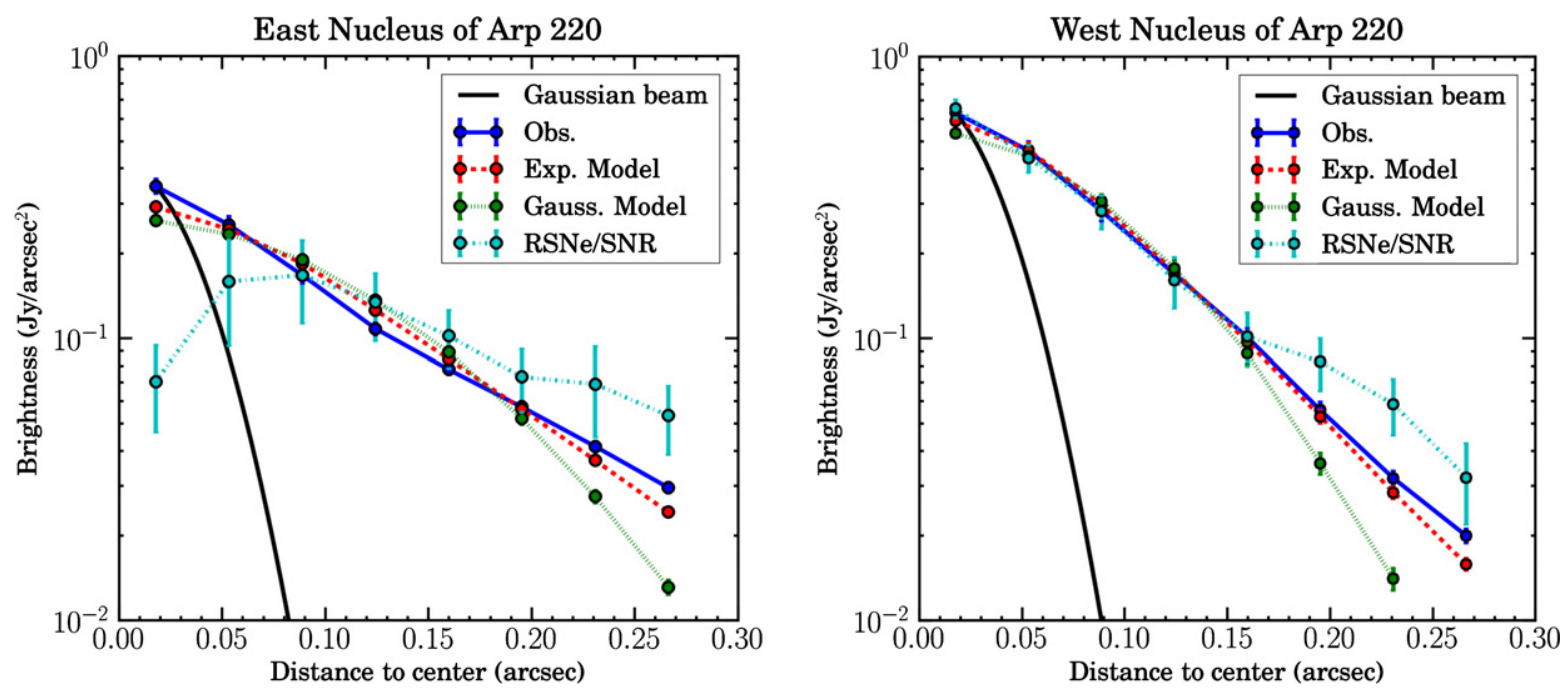

Figure 2. Azimuthally averaged radial profiles for the deprojected image of the east nucleus (left) and west nucleus (right) in semilog space. Blue solid curves are $33 \mathrm{GHz}$ emission, and cyan dotted-dashed line curves are the RSN/SNR number density profiles scaled to match the $33 \mathrm{GHz}$ radial profile at 0 '. 09 . Red dotted lines and green dashed lines are the radial profiles of the exponential and Gaussian model images, respectively, convolved with the beam. The black solid lines represent the Gaussian beam radial profile.

Table 1

Flux Densities and Spectral Indices for Arp 220

\begin{tabular}{|c|c|c|c|c|c|}
\hline \multirow{2}{*}{$\begin{array}{l}\text { Frequency } \\
(\mathrm{GHz})\end{array}$} & \multirow{2}{*}{$\begin{array}{l}\text { Total } \\
\text { (mJy) }\end{array}$} & \multicolumn{2}{|c|}{ East Nucleus } & \multicolumn{2}{|c|}{ West Nucleus } \\
\hline & & Integrated (mJy) & Peak $\left(\mathrm{mJy} \text { beam }^{-1}\right)^{\mathrm{a}}$ & Integrated (mJy) & Peak $\left(\mathrm{mJy} \text { beam }^{-1}\right)^{\mathrm{a}}$ \\
\hline 4.7 & $222.0 \pm 1.9$ & $92.4 \pm 2.1$ & $61.8 \pm 0.5$ & $114.6 \pm 4.3$ & $89.5 \pm 0.7$ \\
\hline 7.2 & $171.4 \pm 2.3$ & $73.2 \pm 1.4$ & $36.0 \pm 0.4$ & $89.5 \pm 1.4$ & $60.4 \pm 0.7$ \\
\hline 5.95 & $197.6 \pm 2.8$ & $81.4 \pm 2.8$ & $49.0 \pm 0.7$ & $94.3 \pm 1.7$ & $73.3 \pm 1.0$ \\
\hline 32.5 & $61.8 \pm 7.2$ & $30.1 \pm 3.9$ & $4.1 \pm 0.5$ & $33.4 \pm 4.0$ & $6.5 \pm 0.8$ \\
\hline \multicolumn{6}{|c|}{ Spectral index } \\
\hline$\alpha_{6-33 \mathrm{GHz}}$ & $-0.69 \pm 0.07$ & $-0.59 \pm 0.08$ & & $-0.61 \pm 0.07$ & \\
\hline$\alpha_{4.7-7.2 \mathrm{GHz}}$ & $-0.61 \pm 0.04$ & $-0.55 \pm 0.07$ & & $-0.58 \pm 0.09$ & \\
\hline
\end{tabular}

Notes. For details on the calculations, see Section 3.1.

a The clean restoring beam FWHM is $0^{\prime \prime} 60 \times 00^{\prime \prime} 43$ at $4.2 \mathrm{GHz}, 0^{\prime \prime} .38 \times 0^{\prime \prime} .28$ at 5.95 and $7.2 \mathrm{GHz}$, and $0^{\prime \prime} 081 \times 0^{\prime \prime} 063$ at $32.5 \mathrm{GHz}$.

$33 \mathrm{GHz}$ phase calibrator. When revised from the nominal VLA position to the position reported in the VLBI calibrator catalog, the astrometric agreement between our image and the other images improved to a fraction of a beam. For reference, in our data the peak positions of the two nuclei at $33 \mathrm{GHz}$ are $\alpha_{2000}=$ $15^{\mathrm{h}} 34^{\mathrm{m}} 57^{\mathrm{s}} .291 \pm 0.003\left( \pm 0^{\prime} .05\right), \delta_{2000}=23^{\circ} 30^{\prime} 11^{\prime \prime} .34 \pm 0^{\prime} .04$ (east) and $\alpha_{2000}=15^{\mathrm{h}} 34^{\mathrm{m}} 57^{\mathrm{s}} .222 \pm 0.002\left( \pm 0^{\prime} .03\right), \delta_{2000}=$ $23^{\circ} 30^{\prime} 11^{\prime \prime} .51 \pm 0^{\prime} .03$ (west). We derive these position via a Gaussian fit (CASA's imf it) but they also closely coincide with the positions of the highest intensity pixel for each nucleus. The uncertainties in the peak positions above may also be viewed as our overall astrometric uncertainty, which we derive from the standard deviation between the positions of the highest intensity pixels from our $6 \mathrm{GHz}$ image and our shifted $33 \mathrm{GHz}$ image, and the archival images from Norris (1988), Condon et al. (1991), Sakamoto et al. (2008), Wilson et al. (2014), and N. Z. Scoville et al. (in preparation).

\section{RESULTS}

In Figures 1 and 2, we present new VLA images of Arp 220 at 6 and $33 \mathrm{GHz}$ and the radial profiles of each nucleus at $33 \mathrm{GHz}$. Using these new data, combined with a $\mathrm{CO}(3 \rightarrow 2)$ integrated intensity ("zeroth moment") map from Sakamoto et al. (2008), and positional information of point sources found by Lonsdale et al. (2006), we carry out a series of calculations to determine what mechanism is producing most of the radio emission, how radio emission traces recent star formation, and the true sizes and shapes of the nuclear disks. In Tables 1-3 we present the results of these calculations.

\subsection{Integrated Flux Densities and Spectral Indices}

In Table 1, we report the flux density of the entire system, each nucleus, and the resulting spectral indices. We used a curve of growth method to derive the flux densities. For the integrated flux density, we progressively $(u, v)$ tapered and re-imaged the data, recording the total flux density above a signal-to-noise of five at each resolution. These flux densities agree with those measured from the imaging of individual arrays (see Section 2). For the flux densities of the individual nuclei, we used CASA's imstat task to place circular apertures around each component, varying their radii. We plotted the flux density against aperture radius and looked for convergence in this curve-of-growth to identify the true flux density. We also independently measured the integrated flux of the southwest component seen at $C$ band (see Figure 1(a)), using an aperture in the CASA viewer, and found that it encloses $\sim 3 \%$ of the total $5.95 \mathrm{GHz}$ flux density. The integrated flux densities at both frequencies agree, within the reported errors, with predicted values based on the modeled 
Table 2

Best-fit Morphology for the Nuclei of Arp 220 at $33 \mathrm{GHz}$

\begin{tabular}{|c|c|c|c|c|}
\hline \multirow[t]{2}{*}{ Parameter } & \multicolumn{2}{|c|}{ East Nucleus } & \multicolumn{2}{|c|}{ West Nucleus } \\
\hline & Deconvolved $^{\mathrm{a}}$ & Convolved $^{\mathrm{b}}$ & Deconvolved $^{\mathrm{a}}$ & Convolved $^{\mathrm{b}}$ \\
\hline \multicolumn{5}{|c|}{ Exponential disk model } \\
\hline Scale length (pc) & $30.3 \pm 4.6$ & $34.0 \pm 5.1$ & $21.0 \pm 3.2$ & $25.4 \pm 3.8$ \\
\hline Peak intensity $\left(\mathrm{mJy}\right.$ beam $\left.^{-1}\right)$ & $6.0 \pm 0.7$ & $4.8 \pm 0.6$ & $13.4 \pm 1.6$ & $9.0 \pm 1.1$ \\
\hline Position angle $\left({ }^{\circ}\right)$ & $54.7 \pm 0.6$ & $55.4 \pm 0.6$ & $79.4 \pm 0.8$ & $77.3 \pm 0.8$ \\
\hline Inclination $\left(^{\circ}\right)$ & $57.9 \pm 0.6$ & $55.4 \pm 0.6$ & $53.5 \pm 0.5$ & $49.1 \pm 0.5$ \\
\hline$R_{50}(\mathrm{pc})^{\mathrm{c}}$ & $50.8 \pm 7.6$ & $57.0 \pm 8.6$ & $35.2 \pm 5.3$ & $42.7 \pm 6.4$ \\
\hline \multicolumn{5}{|c|}{ Two-dimensional Gaussian fitting } \\
\hline FWHM major axis (pc) & $85.9 \pm 8.6$ & $90.8 \pm 9.1$ & $63.7 \pm 6.4$ & $70.2 \pm 7.0$ \\
\hline FWHM minor axis (pc) & $46.3 \pm 4.6$ & $51.8 \pm 5.2$ & $38.0 \pm 3.8$ & $44.7 \pm 4.5$ \\
\hline Position angle $\left({ }^{\circ}\right)$ & $56.0 \pm 1.1$ & $56.5 \pm 1.1$ & $78.7 \pm 1.6$ & $77.1 \pm 1.5$ \\
\hline \multicolumn{5}{|c|}{ Observed half-light radius $^{\mathrm{d}}$} \\
\hline$R_{50 \text { sky }}(\mathrm{pc})$ & & $73.3 \pm 7.5$ & & $45.5 \pm 3.7$ \\
\hline
\end{tabular}

Notes. The reported parameters were obtained by fitting a 2D exponential and Gaussian distribution, respectively. The quoted uncertainties reflect the systematic uncertainty from varying the goodness of fit statistic or other methodology in the fit. In the case of the peak intensity, the uncertainty is determined by the flux density calibrator error, $12 \%$. In all cases, the errors from the fit are negligible. For Arp $220\left(d_{L}=77 \mathrm{Mpc}\right), 10 \mathrm{pc} \approx 0^{\prime} \cdot 03$ or $0^{\prime \prime} 1=36.9 \mathrm{pc}$.

${ }^{a}$ Parameters that construct the best image, compared to the observed one, after convolving the model with the reported clean beam (see Section 3.3 for details).

${ }^{\mathrm{b}}$ Best-fit parameters that reconstruct the observed image without accounting for the beam.

${ }^{c}$ This is an analytical solution obtained by using the scale length parameter from the model. We refer to the deconvolved column as $R_{50 d}$.

d Taking $R_{50 \text { sky }}=\sqrt{A_{50 \text { sky }} /(\pi \cos i)}$, where $i$ is the inclination obtained from the exponential disk model and $A_{50}$ sky is the observed area enclosing half of the total $33 \mathrm{GHz}$ flux density. The effects of the beam are not accounted for in this size metric.

Table 3

Average Brightness Temperatures in Arp 220

\begin{tabular}{lccc}
\hline \hline $\begin{array}{l}\text { Frequency } \\
(\mathrm{GHz})\end{array}$ & $\begin{array}{c}\text { Total System } \\
\log \left(T_{b}(\mathrm{~K})\right)\end{array}$ & $\begin{array}{c}\text { East Nucleus } \\
\log \left(T_{b}(\mathrm{~K})\right)\end{array}$ & $\begin{array}{c}\text { West Nucleus } \\
\log \left(T_{b}(\mathrm{~K})\right)\end{array}$ \\
\hline 5.95 & $4.64 \pm 0.07$ & $4.43 \pm 0.10$ & $4.81 \pm 0.10$ \\
32.5 & $2.7 \pm 0.1$ & $2.5 \pm 0.1$ & $2.9 \pm 0.1$ \\
\hline
\end{tabular}

Notes. Integrated values are calculated within $A_{50 d}$ (deconvolved modeled size) at $33 \mathrm{GHz}$ (more in Section 3.4). The uncertainties follow from propagation of uncertainties quoted earlier in this paper.

integrated SED published in the literature (e.g., Anantharamaiah et al. 2000).

Using these flux densities, we calculated the spectral indices, $\alpha$ for $F_{v} \propto v^{\alpha}$, of the whole system and each nucleus via

$$
\alpha_{1-2}=\frac{\log F_{1}-\log F_{2}}{\log v_{1}-\log v_{2}},
$$

where $F_{1}$ and $F_{2}$ are the flux densities at frequencies $v_{1}$ and $v_{2}$. Equation (1) will be valid for flux densities $F_{1}$ and $F_{2}$ over matched apertures (or for integrated values over whole systems).

\subsection{Comparison to Gas and Recent Star Formation}

To assess the degree to which the measured sizes are characteristic of the whole system, we compared our maps to known distributions of emission from gas and recent radio supernovae and/or supernova remnants (RSNe/SNRs). In Figure 1(b), we plot $C$ band contours over the $\mathrm{CO}(3 \rightarrow 2)$ map of Sakamoto et al. (2008; restoring Gaussian beam with FWHM 0!38 $\times 0$ 0'28 at a p.a. $\approx 23^{\circ}$.

The RSNe/SNRs trace recent star formation, and they can accelerate cosmic ray (CR) electrons that emit synchrotron radiation. We built a map of RSN/SNR number density from the locations of 49 point sources identified by Lonsdale et al. (2006) from $18 \mathrm{~cm}$ VLBI observations. On our $33 \mathrm{GHz}$ astrometric grid, we convolved delta functions with a fixed, fiducial intensity at the positions of the point sources with our $33 \mathrm{GHz}$ beam. ${ }^{14}$ In Figures 1(c) and (d), we compare the $33 \mathrm{GHz}$ map to the distribution of recent RSNe/SNRs.

Note that although we use RSNe/SNRs as signposts of recent star formation, the VLBI sources do not contribute significantly to the flux that we observe. For a typical synchrotron spectral index $\alpha_{1.7-33 \mathrm{GHz}}=-0.7$, the Lonsdale et al. (2006) RSNe/ SNRs would contribute $1.5 \mathrm{mJy}$ at $33 \mathrm{GHz}$ and $4.9 \mathrm{mJy}$ at $6 \mathrm{GHz}$. This contribution would only account for $\sim 2.5 \%$ of the total flux density that we observe with the VLA. Even at $18 \mathrm{~cm}$, the Lonsdale et al. (2006) RSNe/SNRs have integrated flux only $\sim 12 \mathrm{mJy}$ at $18 \mathrm{~cm}$, or $\sim 4 \%$ of the total flux density of Arp 220 at that frequency (Williams \& Bower 2010). This contribution is small compared to the $10 \%$ expected fraction in normal spiral galaxies like M31 or the Milky Way (Pooley 1969;

\footnotetext{
${ }^{14}$ Lonsdale et al. (2006) report offsets from the center of Arp 220, which we
} take to be $\alpha_{2000}=15^{\mathrm{h}} 34^{\mathrm{m}} 57^{\mathrm{s}} .259, \delta_{2000}=23^{\circ} 30^{\prime} 11^{\prime \prime} .409$. 
Ilovaisky \& Lequeux 1972). This difference is most likely due to free-free absorption at $18 \mathrm{~cm}$ (see Section 4.3.4).

\subsection{The Morphology of Arp 220 Nuclei at $33 \mathrm{GHz}$}

The smooth, ellipsoidal isophotes in Figure 1 suggest a disk-like geometry. We modeled the $3 \sigma$ clipped image of Arp 220 at $33 \mathrm{GHz}$ (outermost contour in Figure 1(c)) using a two-dimensional (2D) nonlinear least-squares fitting technique. After experimenting with Gaussian, exponential, Sérsic and hybrid profiles, we found that the two disks are reasonably described by thin, tilted exponential disks. We fit both nuclei simultaneously by varying, without constraints, the amplitude, p.a., inclination, center, and scale length of each nucleus. Although the parameters did not have constraints, the starting points were educated guesses of the final parameters. In each case, we construct the model image, convolve it with the synthesized beam of our observations, and compare the model and observed intensities to derive $\chi^{2}$. Note that the results for the inclination of the disks represent lower limits because we assume thin disks.

The best-fit parameters from the model fitting, along with associated uncertainties, are reported in Table 2. In addition to deriving formal uncertainties, we gauge the accuracy of our fit by varying our approach among several reasonable methods. For example, we adopt a logarithmic, rather than linear, goodness of fit statistic and we fit the radial profile rather than the image itself. These imply an uncertainty of $\approx 15 \%$ for the scale length and a few percent for p.a. and inclination. The error in the normalization is dominated by our overall uncertainty in the amplitude calibration $(\approx 12 \%)$. As another point of comparison, we also report the results of simple Gaussian fitting, although, we emphasize that the residuals are substantially poorer for this approach at low and high radius.

From the exponential model, we obtain deconvolved scale lengths of 30 and $21 \mathrm{pc}$ for the east and west nucleus, respectively. Our results for p.a., inclination, and center did not vary significantly with the choice of functional form. While the fits appear to be good descriptions, they are not perfect. From the residual images, we found that the western nucleus showed higher residuals in the disk than the eastern nucleus, while the center of the eastern nucleus had higher residuals than the western nucleus.

Both nuclei are well resolved, showing significant extent compared with the synthesized beam. The implied deconvolved half-light radii, $R_{50 d}$, are 51 and $35 \mathrm{pc}$, respectively; that is, if viewed face-on, we would expect half the emission from Arp 220 to come from nuclear disks $\sim 100$ (east) and $\sim 70$ (west) pc across. In Figures 1 and 2, we have also shown that the size measurement agrees with that implied by the RSN/SNR distribution (Lonsdale et al. 2006). In fact, Herrero-Illana et al. (2012) derive scale lengths for the RSN/SNR distribution, from Lonsdale et al. (2006) and Parra et al. (2007), that are consistent with the results shown in Table 2 .

The p.a.s of the east and west nuclei agree well with those of the kinematic major axes of the disks measured from submillimeter (sub-mm) CO observations (Sakamoto et al. 1999, 2008), H I absorption observations from Mundell et al. (2001), and $\mathrm{H} 53 \alpha$ radio recombination line Rodríguez-Rico et al. (2005). The velocity gradients along these p.a.s on individual nuclei have also been observed in the $2 \mu \mathrm{m} \mathrm{H} \mathrm{H}_{2}$ line (Genzel et al. 2001) which traces hot molecular gas and $2.3 \mu \mathrm{m} \mathrm{CO}$ absorption (the latter traces stellar velocities: Engel et al. 2011).
We performed two checks on the size measurements. First, as a point of comparison, we report in Table 2 a 2D Gaussian fit to each nucleus at $33 \mathrm{GHz}$. We obtained deconvolved FWHM sizes of 0 '.23 $\times 00^{\prime} .13\left(86 \times 46 \mathrm{pc}^{2}\right)$ for the eastern and $0^{\prime \prime} .17 \times 00^{\prime \prime} 10\left(64 \times 38 \mathrm{pc}^{2}\right)$ for the western nucleus. These sizes agree fairly well with previous, marginally resolved, estimates at other frequencies. Downes \& Eckart (2007) found a deconvolved major axis size of $0{ }^{\prime} .19=70 \mathrm{pc}$ for the western nucleus at $1.3 \mathrm{~mm}$. Sakamoto et al. (2008) found major axis sizes of $0.27=100 \mathrm{pc}$ (FWHM, east) and $0^{\prime} .16=59$ pc (FWHM, west) at $860 \mu \mathrm{m}$. However, we show through radial profiles (Figure 2) that the disks are better described by an exponential morphology. In fact, the deconvolved Gaussian fit would underestimate the deconvolved half-light diameter of the disks by $9 \%$ (west) and $15 \%$ (east), if we account for inclination effect in the Gaussian fit, i.e., the deconvolved FWHM major $_{\text {is smaller }}$ by $\sim 9 \%$ and $15 \%$ when compared to the deconvolved half-light diameter $\left(2 \times R_{50 d}\right)$.

Second, we calculated the area on the sky containing half of the flux associated with each nucleus. This very basic measure still suffers from beam dilution and inclination effects, but provides a measure of size that is independent of the functional form. We derived this image-based $A_{50 \text { sky }}$ by identifying the isointensity contour that encloses $50 \%$ of the total flux density of each nucleus. We summed the area of the pixels (pixel size $=0.02$ ) enclosed within that contour and estimate the observed radius for $i=55^{\circ} .4$ (east) and $i=49^{\circ} .1$ (west), and $R_{50 \text { sky }}=\sqrt{A_{50 \text { sky }} /(\pi \cos i)}$. This is $\sim 73$ pc for the eastern nucleus and $\sim 46 \mathrm{pc}$ for the western nucleus. We report the observed $R_{50 \text { sky }}$ values in Table 2 . As with the Gaussian, the measured area shows broad agreement with the exponential profile fitting, though differing in detail.

We created deprojected, azimuthally averaged radial profiles of $33 \mathrm{GHz}$ intensity to assess the accuracy of our models and compare the structure of the nuclei to that of the RSN/SNR number density map. Assuming a thin tilted ring geometry, we calculated deprojected profiles for the observed emission, the emission in the convolved model, and the RSN/SNR number density map. In each case, the center of the profiles correspond to the highest intensity pixel in the observed image for each nucleus. We plot these profiles in Figure 2. We included only emission above a signal-to-noise ratio of three and then averaged the intensity in a series of inclined, $\sim 0^{\prime} .035$ wide (half the clean beam size) rings, adopting the best-fit model inclination and p.a. from our modeling (Table 2). Note that these rings oversample the $\sim 0^{\prime \prime} 07$ beam, so that adjacent bins in Figure 2 are not independent. We normalized the RSN/SNR radial profile to match the $33 \mathrm{GHz}$ profile at $r \approx 0^{\prime}{ }^{\prime} 09$. The plotted error bars were calculated from the standard deviation of the flux within each annulus divided by the square root of the area in that annulus expressed in units of the beam size (i.e., the number of independent beams).

In Figure 2, we show that the exponential model matches the data well for both nuclei, matching slightly better for the western nucleus. Meanwhile, the Gaussian profile is not as good as the exponential profile when compared to the observed data, being particularly poorer in the outer parts of the disks. The linearity of the semilog profiles also confirms (and motivates) our adoption of an exponential functional form. The RSN/ SNR radial profiles mostly follow the integrated radio emission profiles (and thus also the model). The agreement is better in the western nucleus. The eastern nucleus lacks a bright central peak and shows a somewhat more scattered distribution, which 
could be caused by stochasticity and timescale effects; i.e., there just may not be enough RSNe/SNRs visible to give a smooth appearance (compare to Figure 1(d) to see the clumpy nature of the SNe distribution). The same idea applies for the outskirts of the western nucleus. In the rest of the paper, we will consider that the RSN/SNR number density distribution follow the continuum emission observed at $33 \mathrm{GHz}$ closely enough that we can take our measured $33 \mathrm{GHz}$ sizes as indicative of the distribution of active star formation in Arp 220.

\subsection{Brightness Temperatures}

The brightness temperature, $T_{b}$, can be used to constrain the emission mechanism and energy source, and may give clues regarding the optical depth. With well resolved sizes, we can circumvent beam dilution that often confuses estimates of $T_{b}$. We calculated $T_{b}$ using the Rayleigh-Jeans approximation via

$$
T_{b}=\left(\frac{S_{v}}{\Omega_{\text {source }}}\right) \frac{c^{2}}{2 k_{B} \nu^{2}},
$$

with $S_{v}$ the flux density at frequency $v$ and $\Omega_{\text {source }}$ the area subtended by the source.

We report average Rayleigh-Jeans brightness temperatures, $T_{b}$, in Table 3. From our model, we take the area $A_{50 d} \equiv \pi R_{50 d}^{2}$ that we expect to enclose half the emission if the system were viewed face on (see the "deconvolved" column in Table 2). Assuming this to be the true area of the disk at all frequencies, we derive average $T_{b}$ over the half-light region. This means we used half of the observed flux density for $\mathrm{S}_{v}$ and $A_{50 d}$ for $\Omega_{\text {source }}$ in Equation (2). Our rationale for this assumption is that the $33 \mathrm{GHz}$ image appear to be optically thin, high-resolution tracer of the distribution of recent star formation. Assuming that this structure is common across wavelength regimes allows us to use a "true" size in place of a size observed with a much coarser beam. We also calculated the peak $T_{b}$ at each band from the peak flux density and the area of the clean beam at each frequency. This peak $T_{b}$ is higher at $33 \mathrm{GHz}$ than at $6 \mathrm{GHz}$; this simply reflects that the area of Arp 220 at $33 \mathrm{GHz}$ is smaller than the beam size at $6 \mathrm{GHz}$. For exactly this reason-the small size of Arp 220 and the variable resolution at different frequencies - the peak measurement has limited utility and we only report the "average" version.

The average $T_{b}$ is from $10^{4.4} \mathrm{~K}$ at $6 \mathrm{GHz}$ to $10^{2.5}(\approx 300) \mathrm{K}$ at $33 \mathrm{GHz}$, for the east nucleus and $10^{4.8} \mathrm{~K}$ at $6 \mathrm{GHz}$ to $10^{2.9}$ $(\approx 800) \mathrm{K}$ at $33 \mathrm{GHz}$, for the west nucleus.

\section{DISCUSSION}

Figure 1 shows that our observations clearly separated the nuclei at both 6 and $33 \mathrm{GHz}$ and resolve the structure of both nuclei is resolved at $33 \mathrm{GHz}$. We find a projected nuclear separation of $0^{\prime \prime} .96 \pm 0$. .'01 (354 $\left.\pm 4 \mathrm{pc}\right)$, in agreement with previous works (e.g., Scoville et al. 1998; Soifer et al. 1999; Rodríguez-Rico et al. 2005; Sakamoto et al. 2008). In the following subsections, we discuss the radio continuum emission processes, the correspondence with gas and dust emission, and consequences of the small sizes of the emission regions. There is a large-scale agreement between the locations of radio continuum emission, $\mathrm{CO}$, and RSNe/SNRs, suggesting that the sizes of the radio continuum sources may be viewed as characteristic of the system.

\subsection{Synchrotron Produces Most of the $33 \mathrm{GHz}$ Emission}

Synchrotron radiation appears to produce most of the continuum emission at both 6 and $33 \mathrm{GHz}$. The high brightness temperature of a few $\times 10^{4} \mathrm{~K}$, inferred at $6 \mathrm{GHz}$ by using the $33 \mathrm{GHz}$ nuclear sizes, argues in this direction. This high brightness temperature cannot come from Hir regions, even if they are completely opaque, because in a purely thermal environment, the electron temperature of such regions should not exceed $10^{4} \mathrm{~K}$. If we combined the high brightness temperature with the observed internal $C$ band spectral index of the total system, $\alpha_{4.7-7.2 \mathrm{GHz}}=-0.61 \pm 0.04$, we infer that most of the emission at $6 \mathrm{GHz}$ is synchrotron.

The spectral index between 6 and $33 \mathrm{GHz}, \alpha_{6-33 \mathrm{GHz}}=$ $-0.69 \pm 0.07$, matches the internal $C$ band $\alpha$ of the total system within the errors; the same is true for the two nuclei separately (see Table 1 ). The similarity between $\alpha_{4.7-7.2 \mathrm{GHz}}$ and $\alpha_{6-33 \mathrm{GHz}}$ indicates no significant spectral flattening between 6 and $33 \mathrm{GHz}$, suggesting that synchrotron dominates the emission across this range of frequencies. Reinforcing this point, our total flux density and spectral index agree with the predictions made by Anantharamaiah et al. (2000, see their Figure 10(b)). They found synchrotron emission to dominate below $\sim 60 \mathrm{GHz}$, and estimated the thermal fraction at $6 \mathrm{GHz}$ and $33 \mathrm{GHz}$ to be $\sim 15 \%$ and $35 \%$, respectively. If we assume a thermal fraction of $35 \%$ at $33 \mathrm{GHz}$, a nonthermal spectral index of -0.76 (see Table 9 in Anantharamaiah et al. 2000), and a typical thermal spectra index of -0.1 , we obtain $\alpha_{6-33 \mathrm{GHz}} \approx$ -0.60 and $\alpha_{4.7-7.2 \mathrm{GHz}} \approx-0.66$, which deviate $\sim 1.3 \sigma$ from the observed values. The difference between the predicted spectral indices is 0.06 , while for the observed values it is closer to $-0.08 \pm 0.11$. If we assume the observed values are the true ones, this slight discrepancy could indicate potential opacity effects between 4.7 and $7.2 \mathrm{GHz}$ (see Section 4.3.4), or the presence of a steeper nonthermal spectral index between 6 and $33 \mathrm{GHz}$. However, this discrepancy does not affect our interpretation of nonthermal emission dominating at $33 \mathrm{GHz}$. In fact, a higher thermal fraction at $33 \mathrm{GHz}$ will only make this discrepancy worse (see below). Overall, our results are consistent with previous results showing lower thermal fractions at $33 \mathrm{GHz}$ for merging starbursts compared to normal galaxies (Murphy 2013).

The overall SFR of the system provides an alternate way to estimate the expected thermal radio continuum emission. Beginning with the IR $(8-1000 \mu \mathrm{m})$ luminosity of Arp 220, we estimate the expected thermal luminosity of the system if the IR is all due to star formation by following SFR conversions from Table 8 in Murphy et al. (2012) $)^{15}$ and assuming an electron temperature of $7500 \mathrm{~K}$ (Anantharamaiah et al. 2000). This approach predicts a thermal fraction of $\sim 55 \%$ at $33 \mathrm{GHz}$ and $\sim 20 \%$ at $6 \mathrm{GHz}$. This is in good agreement with the thermal fraction at $33 \mathrm{GHz}$ obtained in Condon (1992) for a prototypical starburst, M82. However, if we derive the expected spectral index as we did at the end of the previous paragraph (assuming $55 \%$ of thermal fraction at $33 \mathrm{GHz}$ ), we obtain $\alpha_{6-33 \mathrm{GHz}} \approx-0.49$ and $\alpha_{4.7-7.2 \mathrm{GHz}} \approx-0.57$, which deviates considerably from what we observe between 6 and $33 \mathrm{GHz}$. Note that $\alpha_{4.7-7.2 \mathrm{GHz}}$ does not vary significantly from what we observe, this is due to the small thermal fraction expected at this frequency range.

\footnotetext{
15 Murphy et al. (2012) uses a Kroupa initial mass function (IMF) to derive the theoretical SFR conversions. The operation is equivalent to using such an IMF to relate the ionizing photon production (traced by thermal radio emission) to bolometric luminosity (traced by IR).
} 
The easiest explanation for the lower-than-expected thermal flux is that a significant fraction of the ionizing photons produced by young stars are absorbed by dust before they produce ionizations. This would lower the free-free estimate in the calculation. We estimate that to match our observations, we would require that at least $20 \%$ of the ionizing photons be absorbed by dust. This number seems plausible for an environment as dust embedded as Arp 220 (see Section 4.3.4) and is consistent with some of the arguments made when considering the apparent deficit of IR cooling line emission (Díaz-Santos et al. 2013, and references therein). Alternatively, an initial mass function (IMF) that produces more bolometric light (and thus IR and likely $\mathrm{SNe}$ ) relative to ionizing photons could resolve the discrepancy. That is, we could invoke an "intermediate-heavy" IMF compared to that used in Murphy et al. (2012). We could also reconcile our two estimates if the synchrotron spectral slope decays drastically between 6 and $33 \mathrm{GHz}$, so that the apparent $33-6 \mathrm{GHz}$ index is a combination of very steep, curving synchrotron and emerging thermal emission. However, this would require that thermal emission make up most of the SED at higher frequencies, which is not observed (see Anantharamaiah et al. 2000; Clemens et al. 2010).

Our best interpretation of the data is that the $33 \mathrm{GHz}$ emission is mostly synchrotron, in mild contrast with a typical starburst galaxy (see Figure 1 in Condon 1992). We suggest that the most likely cause is the suppression of thermal radio emission as dust absorbs ionizing photons.

\subsection{The Radio Emission Coincides with Gas, Hot Dust, and RSN/SNR}

In Figure 1(b), we show the $6 \mathrm{GHz}$ emission is largely cospatial with $\mathrm{CO}$ emission. The $6 \mathrm{GHz}$ emission is our more sensitive band, with a beam nearly matched to the $\mathrm{CO}$, and-as just discussed-we expect that it traces the same synchrotron emission as the $33 \mathrm{GHz}$. The $\mathrm{CO}$ and $6 \mathrm{GHz}$ emission cover roughly the same area, have broadly coincident peaks, and both show an extended faint feature to the southwest (Mazzarella et al. 1992, note a similar coincidence between $18 \mathrm{~cm}$ emission and the Arp 220 starburst traced in the near-IR). The distributions of $\mathrm{CO}$ and $6 \mathrm{GHz}$ emission do significantly differ in detail. The ratio of fluxes for the two nuclei is $1: 2$ (east:west) for $\mathrm{CO}$ and almost 1:1 for continuum. In the west nucleus, the morphology is more centrally concentrated at $6 \mathrm{GHz}$ compared to the $\mathrm{CO}$ map. Some of these differences may reflect real differences between the current gas reservoir and recent star formation, but they may also reflect temperature and optical depth effects. The CO $(3 \rightarrow 2)$ emission in this region shows good evidence for optical thickness (Sakamoto et al. 2008), and the densities are high enough that the gas temperature will be likely coupled to the dust $(\sim 100 \mathrm{~K})$. Therefore, making a straightforward interpretation of the $\mathrm{CO}$ in terms of column density is challenging. We draw the broad conclusion from Figure 1(b) that the synchrotron originates from the same region as, and in very rough proportion to, the molecular gas supply.

A similar situation is also observed on smaller spatial scales by the comparison of the RSN/SNR number density map to the $33 \mathrm{GHz}$ map (see Figure 1(d)). The distributions are co-spatial, but the continuum map appears smoother than the map made from individual RSN/SNR. This is particularly evident in the eastern nucleus, where the covering factor of point sources observed with the Very Long Baseline Array (VLBA) is small-perhaps a result of stochasticity in the rate and lifetime of $\mathrm{SN}$ visible using
VLBI measurements. The radial profiles in Figure 2 highlight the quantitative agreement between the continuum and the RSNe/SNRs distribution even more. After azimuthal averaging, the VLBA point source maps are a fairly close match to the $33 \mathrm{GHz}$ continuum. This is also supported by the agreement between the scale lengths reported in Table 2 and those found by Herrero-Illana et al. (2012) based on the radial profiles of the RSNe/SNRs distribution observed by Lonsdale et al. (2006) and Parra et al. (2007).

Such a close match between the $33 \mathrm{GHz}$ continuum extent and the RSN/SNR number density map may not be too surprising: if synchrotron radiation arises from $\mathrm{CR}$ electrons accelerated by $\mathrm{SN}$ shocks, then the $33 \mathrm{GHz}$ continuum emission might be expected to resemble a "puffed up" version of the RSN/SNR distribution due to the diffusion of $\mathrm{CR}$ electrons. Instead the distributions match quite well, consistent with most of the $33 \mathrm{GHz}$ emission coming from very close to the original RSN/SNR and little diffusion or secondary CR electron production. This lack of significant propagation could be explained by the cooling timescales being much smaller than the diffusion time. This is expected in compact starbursts with magnetic fields of the order of a $\mathrm{mG}$ (see measurements from Robishaw et al. 2008; McBride et al. 2014, based on Zeeman splitting of $\mathrm{OH}$ megamaser emission), like Arp 220 (see Figure 1 in Murphy 2009), though not in normal galaxies (Murphy et al. 2006). For Arp 220, the cooling time of CR electrons at $33 \mathrm{GHz}$ is $\sim 10^{3} \mathrm{yr}$, which is a combination of synchrotron, bremsstrahlung, ionization, and inverse Compton losses. We make use of Equation (7) in Murphy (2009) for CR electrons with energies greater than $1 \mathrm{GeV}$ to estimate that the synchrotron emitting electrons at $33 \mathrm{GHz}$ only have time to propagate about $5 \mathrm{pc}$, which is about $1 / 10$ th of the size that we have measured for the nuclei. This short diffusion scale yields a synchrotron image that looks very similar to the sites of original CR production (the RSN/SNR) and thus the sites of active star formation. The advantage of the VLA continuum in this case is that in exchange for coarser native resolution, we achieve sensitivity to most of the flux and spatial scales of interest (and potentially still probe a longer timescale).

The similarity of $6 \mathrm{GHz}, 33 \mathrm{GHz}, \mathrm{CO}$ surface brightness, and the RSN/SNR number density distributions lead us to view our $33 \mathrm{GHz}$ measurement as indicative of the true size of the main disks of star formation and, presumably, gas and hot dust. These morphologies are also consistent with the nuclear morphologies measured in mid-IR with the Keck Telescope (Soifer et al. 1999). Perhaps surprisingly, the two disks appear fairly similar in terms of profile, scale length, and observed flux. The western nucleus appears hotter and more compact but the differences are small factors, not an order of magnitude. The physical interpretation of such similarities is unclear. Possible explanations include a similarity in the progenitors, or some "loss of memory" during the process of funneling gas to the center of the galaxies during the ongoing interaction.

\subsection{The Nuclear Disks are the Most Extreme Starburst Environments in the Local Universe}

\subsubsection{Gas Surface Densities}

Current best estimates of the dynamical mass per nucleus are $\sim 6 \times 10^{9} M_{\odot}$ within $\sim 100$ pc of each nucleus (Engel et al. 2011). These values are still uncertain, with $\sim 2 \times 10^{9} M_{\odot}$ representing a likely lower limit in both nuclei (Engel et al. 2011). The dynamical mass represents an upper limit on the 
gas content. Based on dynamical modeling and CO imaging, Downes \& Solomon (1998) estimated the gas content at $1.1 \times 10^{9}$ and $0.6 \times 10^{9} M_{\odot}$ for the eastern and western nucleus, but embedded in a larger gas disk with total mass $\sim 3 \times 10^{9} M_{\odot}$ (see also Sakamoto et al. 1999, 2008; Downes \& Eckart 2007). These estimates mix dynamical modeling with observations of low- $J$ CO line (up to $J=3 \rightarrow 2$ ) measurements that are likely very optically thick in Arp 220. Papadopoulos et al. (2012) provide an alternative, but unresolved, estimate by focusing on higher $J \mathrm{CO}$ transitions and high critical density tracers (e.g., HCN) to estimate a total molecular gas mass of $\sim(15-28) \times 10^{9} M_{\odot}$ for the entire system. The difficulty with this estimate is apportioning this gas mass to the various components of the system. We consider a conservative approach to be the following: we assume that half of the total molecular gas mass is equally distributed between the two nuclei and the other half in an outer disk (e.g., see Sakamoto et al. 1999, for evidence of an outer disk). This implies $\sim 3.5-7 \times 10^{9} M_{\odot}$ of gas per nucleus from Papadopoulos et al. (2012). This remains in moderate tension with the dynamical masses because it would imply very high gas fractions, but given the mismatch in scales (the dynamical masses are estimated on $\sim 100 \mathrm{pc}$ scales) and uncertainties in modeling, a factor of $\sim 3-5$ uncertainty seems plausible (Sakamoto et al. 2008).

The areas that we measure for the Arp 220 nuclei are stunningly small, especially when compared to the integrated properties of the system. We adopt the literature gas mass of the nuclei as $3.5_{-1.5}^{+3.5} \times 10^{9} M_{\odot}$, with the lower bound set by the Engel et al. (2011) values, the upper bound set by Papadopoulos et al. (2012), and the best estimate consistent (with modest tension) with the latter. We further expect half of the gas mass of each to be distributed within the half-light deconvolved, faceon, area $\left(A_{50 d}\right)$ of our radio images (these trace star formation, so we implicitly assume that gas and star formation track one another within the system). We thus compare $\sim 1.75_{-0.75}^{+1.75} \times$ $10^{9} M_{\odot}$ to our half-light areas to estimate average, nuclear, gas surface densities. The deprojected, deconvolved gas surface densities are $2.2_{-1.0}^{+2.1} \times 10^{5} M_{\odot} \mathrm{pc}^{-2}$ (east) and $4.5_{-1.9}^{+4.5} \times 10^{5}$ (west) $M_{\odot} \mathrm{pc}^{-2}$. These translate to an average, nuclear, total hydrogen column densities of $2.7_{-1.2}^{+2.7} \times 10^{25} \mathrm{~cm}^{-2}$ (east) and $5.6_{-2.4}^{+5.5} \times 10^{25} \mathrm{~cm}^{-2}$ (west) (divide these numbers by 2 for $\mathrm{H}_{2}$ column densities). These nuclear hydrogen columns are 3-4 orders of magnitude higher than those derived from X-ray observations (e.g., Clements et al. 2002; Iwasawa et al. 2005), but they roughly agree with those derived from observations at $860 \mu \mathrm{m}$ (Sakamoto et al. 2008) and $434 \mu \mathrm{m}$ (Wilson et al. 2014). The gas surface densities that we derive roughly resemble the maximum stellar surface density of $\sim 10^{5} M_{\odot} \mathrm{pc}^{-2}$ found in a compilation of literature data by Hopkins et al. (2010). Given the large uncertainty in our mass estimate (and the scatter in the Hopkins et al. 2010 compilation) Arp 220 appears consistent with producing such a "maximal" stellar surface density system. This is especially true when one considers that feedback and further evolution of the system may reduce the efficiency (final fraction of gas converted to stars) in the nuclei below unity (a factor of $\sim 1 / 3$ would produce excellent agreement).

We do not know the thickness of the disks, but by adopting a spherical geometry we can calculate a lower limit to the $\mathrm{H}_{2}$ particle densities in the nuclei. ${ }^{16}$ This is $3.8_{-1.6}^{+3.8} \times$

\footnotetext{
16 The correction to obtain the mass inside a sphere of radius $R_{50 d}$ is larger than the areal correction. For simplicity, we adopt the correction appropriate for a Gaussian, so that the mass within $R_{50 d}$ is $\approx 1 / 3.4$ of the total mass.
}

$10^{4} \mathrm{~cm}^{-3}$ (east) and $11_{-4.5}^{+12} \times 10^{4} \mathrm{~cm}^{-3}$ (west). For comparison, a typical Milky Way molecular cloud has a surface density $\sim 100 M_{\odot} \mathrm{pc}^{-2}\left(N(\mathrm{H}) \sim 10^{22} \mathrm{~cm}^{-2}\right)$ and average particle density $n_{\mathrm{H}_{2}} \sim 100 \mathrm{~cm}^{-3}$. In addition to faster free fall times, correspondingly more efficient star formation, and phenomenal opacity, potential implications of such high molecular gas densities would include the secondary production of CR electrons and confinement of CR electrons.

\subsubsection{Infrared Surface Densities and Star Formation Rates}

By following the same approach, we assume the IR emission in Arp 220 is coincident within our measured radio distribution and explore the implications. Conventionally, the IR luminosity surface density, $\Sigma_{\mathrm{IR}}$, is defined as the luminosity per unit area of the system. We calculate $\Sigma_{\mathrm{IR}}$ by assuming that half of the IR luminosity (from 8 to $1000 \mu \mathrm{m}$ ) is generated within the deconvolved, face-on, half-light area $\left(A_{50}\right)$, i.e., we calculate an average IR luminosity surface density, within the half-light area, via

$$
\Sigma_{\mathrm{IR}}=\left(\frac{0.5 \times L_{\mathrm{IR}}[8-1000 \mu \mathrm{m}]}{A_{50}}\right)=\left(\frac{L_{50}}{A_{50}}\right) .
$$

Following our measurements above, we use $A_{50 d} \equiv \pi\left(R_{50 d \text {, east }}^{2}{ }^{+}\right.$ $R_{50 d \text {,west }}^{2}$ ) to derive a total (face-on) IR luminosity surface density of $\Sigma_{\mathrm{IR}} \sim 6.0_{-1.5}^{+2.3} \times 10^{13} L_{\odot} \mathrm{kpc}^{-2}$. ${ }^{17}$ If we further assume that the ratio of the fluxes between the east and west nuclei at $33 \mathrm{GHz}(\sim 1: 1)$ holds at IR wavelengths, then using the derived radio $A_{50 d} \equiv \pi R_{50 d}^{2}$ for the individual disks, we obtain $\Sigma_{\mathrm{IR}} \sim$ $4.2_{-0.7}^{+1.6} \times 10^{13} L_{\odot} \mathrm{kpc}^{-2}$ and $\Sigma_{\mathrm{IR}} \sim 9.7_{-2.4}^{+3.7} \times 10^{13} L_{\odot} \mathrm{kpc}^{-2}$ for the east and west nucleus, respectively. These values are more than an order of magnitude higher than those for the central $0.3 \mathrm{pc}$ of the Orion Nebula complex and M $82(\sim 2 \times$ $10^{12} L_{\odot} \mathrm{kpc}^{-2}$ and $\sim 9 \times 10^{11} L_{\odot} \mathrm{kpc}^{-2}$, respectively (Soifer et al. 2000)), but are closer to those found in the brightest clusters within starburst galaxies $\left(\sim 5 \times 10^{13} L_{\odot} \mathrm{kpc}^{-2}\right.$; Meurer et al. 1997). Our estimated surface densities are consistent with Soifer et al. (2000), who estimated IR luminosity surface densities of $1-6 \times 10^{13} L_{\odot} \mathrm{kpc}^{-2}$ based on mid-IR Keck observations and radio data from Condon et al. (1991).

The deprojected SFR surface density (defined as the SFR per unit area in the disk), $\Sigma_{\mathrm{SFR}}$, is a close corollary of the IR luminosity surface density. We estimate this quantity within the deconvolved, face-on, $A_{50 d}$ for each nucleus at $33 \mathrm{GHz}$, using a 1:1 ratio between east and west, the radio luminosity to SFR conversion from Table 8 in Murphy et al. (2012), and an electron temperature and nonthermal spectral index of $7500 \mathrm{~K}$ and 0.76 (the spectral index in Murphy et al. 2012 is defined with the opposite sign compared to our definition), respectively, from Anantharamaiah et al. (2000). We obtain a $\Sigma_{\text {SFR }}$ of $\sim 10^{3.7 \pm 0.1}$ and $10^{4.1 \pm 0.1} M_{\odot} \mathrm{yr}^{-1} \mathrm{kpc}^{-2}$ within the half-light of the eastern and western nuclei, respectively (divide these numbers by two to take into account both sides of the disks). The total SFR calculated from $L_{\mathrm{IR}}, 180 M_{\odot} \mathrm{yr}^{-1}$, and from the total radio flux density at $33 \mathrm{GHz}\left(L_{\text {Radio }}\right), 195 M_{\odot} \mathrm{yr}^{-1}$, differ by only $\sim 10 \%$, consistent with Arp 220 lying on the $(33 \mathrm{GHz})$ radio-to-far IR correlation (and meaning that we would obtain essentially the same $\Sigma_{\text {SFR }}$ for either luminosity). The radio SFR value differs by $20 \%$ from that derived from Anantharamaiah et al. (2000), which we consider to be within the uncertainties of such calculations.

\footnotetext{
17 The uncertainties in this value, and in the rest of this section, correspond to
} the errors associated with $R_{50 d}$. 
$\Sigma_{\text {IR }}$ tells us coarsely about the density of IR luminosity per unit area, but not necessarily the flux at the surface of the source, which may have important implications for feedback and depends on the detailed geometry of the system. A spherical geometry provides a useful limit on the flux at the surface of the system. In this case, $F_{\text {sphere }}=L_{50} /\left(4 \pi R_{50 d}^{2}\right)$ will be the flux at the surface of a sphere of radius $R_{50 d}$ with the luminosity of Arp 220. Using the measured radio sizes, $F_{\text {sphere }} \sim 1.5_{-0.4}^{+0.6} \times$ $10^{13} L_{\odot} \mathrm{kpc}^{-2}$ for the entire system, $\sim 1.1_{-0.2}^{+0.4} \times 10^{13} L_{\odot} \mathrm{kpc}^{-2}$ for the eastern nucleus and $\sim 2.4_{-0.6}^{+0.9} \times 10^{13} L_{\odot} \mathrm{kpc}^{-2}$ for the western nucleus. A less extreme case, one that may well apply to Arp 220, is a two-sided disk. With one half of the luminosity emergent from each side, we have $F_{\text {disk }}=L_{50} /\left(2 \pi R_{50 d}^{2}\right)$, twice the spherical case. As one would expect, these values are lower than the simple $\Sigma_{\mathrm{IR}}$, but they also differ from one another, reinforcing the importance of geometry to the physics of the source. ${ }^{18}$

Yet another subtlety arises in the specific case where one wishes to calculate the flux through an area very close to, but just above one side of a disk. This quantity is relevant to the oftendiscussed case of radiation pressure on dust (see Section 4.3.3) but because of projection effects it is not identical to any of the above quantities. In Appendix A, we show that this onesided flux perpendicular to the disk, which we call $F_{\text {near }}$ is equal to $L_{\mathrm{IR}} /\left(8 \pi R_{50 d}^{2}\right)$ (Equation (A7)) in general. This value is further divided by an extra factor of two for the case of the two nuclei of Arp 220 (because $L_{\mathrm{IR}}$ combines the light from the two nuclei). From this calculation, we obtain $F_{\text {near }} \sim 1.5_{-0.4}^{+0.6} \times$ $10^{13} L_{\odot} \mathrm{kpc}^{-2}$ for the entire system (the same as for $F_{\text {sphere }}$, though not for the same reason), $\sim 1.1_{-0.2}^{+0.4} \times 10^{13} L_{\odot} \mathrm{kpc}^{-2}$ for the east nucleus and $\sim 2.4_{-0.6}^{+1.0} \times 10^{13} L_{\odot} \mathrm{kpc}^{-2}$ for the west nucleus. As discussed in Appendix A, these should be the most appropriate fluxes to consider when assessing the impact of pressure from radiation perpendicular to the disk.

The ratio of the flux densities between the east and west nuclei varies with frequency. Some other ratios for the east:west relation found in the literature include 1:4 at mid-IR (Soifer et al. 1999), $1: 3$ at $18 \mathrm{~cm}$ (if we consider only the contribution of the point sources from Lonsdale et al. 2006) and 1:2 at sub-mm wavelengths (Sakamoto et al. 2008). However, most of these observations do not offer high enough spatial resolution to truly isolate the contribution of each nucleus. If we ignore this issue and we assume a ratio of $1: 4$, and we use Equation (A7), we obtain $F_{\text {near,east }} \sim 4.5_{-1.1}^{+1.7} \times 10^{12} L_{\odot} \mathrm{kpc}^{-2}$ and $F_{\text {near,west }} \sim 3.7_{-0.9}^{+1.4} \times 10^{13} L_{\odot} \mathrm{kpc}^{-2}$. In every case, we obtain $F_{\text {near }} \gtrsim 10^{13} L_{\odot} \mathrm{kpc}^{-2}$ for the west nucleus, which is always the higher intensity nucleus.

Note that the same geometric issues discussed here raise a caveat regarding the luminosity of the source, which was derived under the assumption of isotropic emission (Sanders et al. 2003; Sanders \& Mirabel 1996). An optically thick thin disk is not an isotropic emitter. However, neither do our observations constrain the geometry of the IR photosphere, which is the relevant surface for this calculation. We discuss this issue in Appendix A. Lacking information, we have assumed the isotropic luminosity throughout this paper, but note the uncertainty (see also Downes \& Eckart 2007; Wilson et al. 2014). Note that high angular

\footnotetext{
18 Optical depth will provide an additional complication. Here we consider the IR surface brightness near the ultimate source of the luminosity in the region of active star formation. As the radiation scatters out of the system, the geometry may change, so that the geometry of the photosphere could differ from the central source considered here.
}

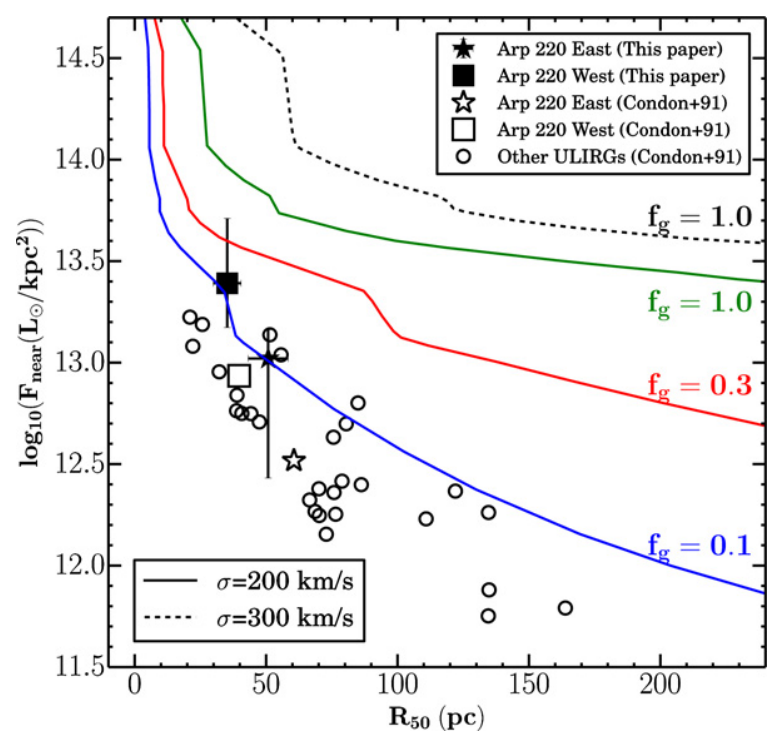

Figure 3. Flux vs. radius for local ULIRGs from Condon et al. (1991; open symbols), and including the values for the eastern (star symbol) and western (square symbol) nuclei of Arp 220 from this paper (filled symbols). In this figure, we represent a new version of Figure 4 of Thompson et al. (2005). The $y$ axis corresponds to the flux passing through a surface area near the source, $F_{\text {near }}$ (see Appendix A), and the $x$ axis to the deconvolved half-light radius. The solid lines represent the Eddington limits for several different gas fractions, $f_{\mathrm{g}}$, assuming stellar velocity dispersion of $\sigma=200 \mathrm{~km} \mathrm{~s}^{-1}$. The dashed line shows the model for $f_{\mathrm{g}}=1$ and $\sigma=300 \mathrm{~km} \mathrm{~s}^{-1}$. From this figure, we observe that Arp 220 appears among the brightest systems but still well below a conservative dusty Eddington limit described by the green line.

resolution IR (from 8 to $1000 \mu \mathrm{m}$ ) observations are needed in order to better constrain the true morphology of the IR photosphere of Arp 220.

\subsubsection{Radiation Pressure and Maximal Starburst Models}

Scoville (2003) and Thompson et al. (2005) argued that for optically thick, dense starburst galaxies, the critical feedback mechanism acting against gravitational collapse, and thus star formation, could be radiation pressure on dust. Although there is ongoing debate about whether or not radiation pressure on dust represents the dominant feedback mechanism in compact starbursts (see Krumholz \& Thompson 2013; Socrates \& Sironi 2013; Davis et al. 2014, for further discussion), the maximal starburst model of Scoville (2003) and Thompson et al. (2005) represents an interesting point of comparison for our present work. ${ }^{19}$ In Figure 3, which closely follows Figure 4 of Thompson et al. (2005), we present our new measurements for the flux near the surface of the source (assuming a thin disk geometry, see Section 4.3.2 and Appendix A) in the context of literature observations and predictions by Thompson et al. (2005). The literature observations show a sample of ULIRGs with sizes based on $8.44 \mathrm{GHz}$ radio maps by (Condon et al. 1991) and luminosities from IRAS. Following the approach presented in Appendix A, we calculate $F_{\text {near }}$ following Equation (A7) assuming half of the total IR luminosity to be enclosed within $A_{50}=\pi R_{50}^{2}$, where $R_{50}=b_{\text {maj }} / 2$. We used the deconvolved FWHM major axis, $b_{\text {maj }}$, from Condon et al. (1991) in order to account for inclination effects (we only include resolved sources). Following the discussion in the previous section, the

\footnotetext{
19 In addition to radiation pressure, cosmic ray pressure has been forwarded as a potentially important feedback mechanism in compact starbursts (Socrates et al. 2008).
} 
fluxes for the points in Figure 3 differs by a factor of eight compared to those in Thompson et al. (2005), ${ }^{20}$ for reasons discussed in Appendix A.

The error bars of our Arp 220 values (filled points in Figure 3) correspond to a combination of the uncertainties in $R_{50 d}$, and uncertainty in the contribution of each nucleus to the IR luminosity, with the lower/upper limit assuming a ratio of 1:4 between east and west (Soifer et al. 1999). To be conservative, we also assume a $20 \%$ uncertainty in the assumption that half of the total IR luminosity is coming from $A_{50}$. The plotted values for the flux correspond to $F_{\text {near,east }} \sim 1.1_{-0.8}^{+0.4} \times 10^{13} L_{\odot} \mathrm{kpc}^{-2}$ and $F_{\text {near,west }} \sim 2.4_{-0.9}^{+2.7} \times 10^{13} L_{\odot} \mathrm{kpc}^{-2}$. In this figure, we show the "Eddington" values for radiation pressure on dust as solid and dashed lines. These represent an envelope for which radiation pressure on dust balances self-gravity. As a result, no equilibrium star-forming system is expected to exist above this line, hence the "Eddington limit" analogy.

The precise value of the limit depends on the size, gas fraction $\left(f_{\mathrm{g}}\right)$ stellar velocity dispersion $(\sigma)$, Rosseland mean opacity $(\kappa)$, and dust-to-gas ratio of the system, leading to the large spread in the model lines seen in the figure. Our measurements of Arp 220 appear as solid points in Figure 3. There, the west nucleus of Arp 220 appears among the highest brightness systems. However, the west nucleus does not clearly stand out from the other ULIRGs with respect to this value, partially because the more compact size means that the "maximal" value is larger for Arp 220 than for larger systems. Overall, all the systems plotted in Figure 3 lie roughly around the Eddington limit for a $f_{\mathrm{g}}=0.1$ and $\sigma=200 \mathrm{~km} \mathrm{~s}^{-1}$ disk in Thompson et al. (2005), which is indicated by the blue solid line.

If we adopt $f_{\mathrm{g}}=1$ and $\sigma \approx 200 \mathrm{~km} \mathrm{~s}^{-1}$ (e.g., Genzel et al. 2001 ), indicated by the green line in Figure 3 , then we calculate a conservative Eddington limit of $\sim 9 \times 10^{13} L_{\odot} \mathrm{kpc}^{-2}$ for the west nucleus and $\sim 7 \times 10^{13} L_{\odot} \mathrm{kpc}^{-2}$ for the east nucleus. Refined measurements of the geometry, gas fraction, opacity, dust-togas ratio, and kinematics are needed to specify the models more precisely. For most plausible assumed disk properties, we can say that both Arp 220 nuclei lie well below the Thompson et al. (2005) Eddington-limited starburst value, with the western nucleus being the brightest system among the local ULIRGs. If improved measurements demonstrate one or both nuclei to lie significantly above this value, one would need to consider luminosity sources other than star formation (presumably an active galactic nucleus (AGN); Iwasawa et al. 2005; Downes \& Eckart 2007; Rangwala et al. 2011) or question the basic assumptions about geometry and equilibrium embedded in the model, but at present little such tension appears to exist.

Another way to assess the role of radiation pressure in Arp 220 is to assume that radiation pressure does represent the dominant force acting against gravity (see Appendix B) and to calculate the required gas opacity, $\kappa$, of the system in order to be in hydrostatic equilibrium. By following Equation (B2), and using the derived values for the gas surface density and the flux for each nucleus (see Sections 4.3.1 and 4.3.2), we find that $\kappa_{\text {east }} \approx 300 \mathrm{~cm}^{2} \mathrm{~g}^{-1}$ and $\kappa_{\text {west }} \approx 80 \mathrm{~cm}^{2} \mathrm{~g}^{-1}$ would be required for radiation pressure to balance gravity. Compared to the models from Semenov et al.

\footnotetext{
20 This factor of eight increases for systems having more than one component, in which case we also divide the total flux among the components. For example, in the case of one individual region in Arp 299, NGC 3690, the difference is an additional factor of $\sim 4$ that comes from the contribution of that region to the total IR luminosity of the system (Alonso-Herrero et al. 2000). For the other systems having more than one component, we used the relative contribution of each component to the integrated flux density observed at $8.44 \mathrm{GHz}$ as a template for the relative contribution at IR wavelengths.
}

(2003), which were used in the models from Thompson et al. (2005), these appear to be unrealistically high values for the gas opacity. We interpret these high values as a reinforcement of our previous findings that the nuclei of Arp 220 lie below the dusty Eddington limit for $f_{\mathrm{g}} \sim 1$ and $\sigma=200 \mathrm{~km} \mathrm{~s}^{-1}$ by a factor of $\sim 10$, and then are not radiation pressure supported.

\subsubsection{Optical Depth}

The small sizes of the Arp 220 nuclei also imply that optical depth effects will be important across the spectrum, even at wavelengths as long as sub-mm. By following the same approach described in Section 3.4, we calculate the average Rayleigh-Jeans brightness temperatures that would be implied by combining the deconvolved half-light sizes of the nuclei with half of the $860 \mu \mathrm{m}$ continuum flux densities reported by Sakamoto et al. (2008) from lower resolution observations. At this wavelength, we find implied average brightness temperatures of $19 \mathrm{~K}$ (east) and $76 \mathrm{~K}$ (west), within the deconvolved half-light area calculated in this paper $\left(A_{50 d}\right)$, without any accounting for optical depth (which could change the size of the apparent emission). In the west nucleus, this approaches the apparent dust temperature of $~ 90 \mathrm{~K}$ (see González-Alfonso et al. 2012, for a more thorough idea of the dust temperature in Arp 220), so that optical depth must become important by this wavelength regime, with $\tau \gtrsim 1$ by $860 \mu \mathrm{m}$ in the western nucleus. This neglects any more complicated geometric considerations (e.g., see the discussion of an inclined geometry in Downes \& Eckart 2007), which are likely to make the situation even more confused. Optical depth effects appear less severe by $\lambda \sim 1 \mathrm{~mm}$, combining $A_{50 d}$ with half of the western nucleus dust emission from Downes \& Eckart (2007), implies an average $T_{\mathrm{b}}^{1 \mathrm{~mm}} \sim 45 \mathrm{~K}$ within the half-light area. ${ }^{21}$ This modest millimeter optical depth is consistent with measurement of a spectral index steeper than $\alpha=2$ by Sakamoto et al. (2008), implying somewhat optically thin emission.

Even this simple calculation demonstrates that by sub-mm wavelengths optical depth effects cannot be neglected, especially in the western nucleus. As a result, we would expect high-resolution but higher frequency observations, e.g., at submm wavelengths with ALMA, to observe a moderately optically thick "photosphere" around the galaxy and so recover a larger size than we find here (e.g., see the continuum observations by Wilson et al. 2014). Similarly, line observations at these wavelengths will need to consider the effects of a moderately optically thick sub-mm continuum in their interpretation.

Furthermore, we can estimate the optical depth at $33 \mathrm{GHz}$. The brightness temperature calculated for the entire source at $33 \mathrm{GHz}$ is $\sim 500 \mathrm{~K}$ (see Table 3 ). The thermal fraction at this frequency is $\sim 35 \%$, so that $T_{b}$ of the thermal emission is $\sim 175 \mathrm{~K}$. The thermal electron temperature $\left(T_{e}\right)$ cannot exceed $\approx 10^{4} \mathrm{~K}$ because line cooling is high at such high temperatures. Then taking $T_{e} \sim 10^{4} \mathrm{~K}$ and a measured $\sim 175 \mathrm{~K}$ brightness, we estimate the average $33 \mathrm{GHz}$ free-free opacity within the half-light radius to be $\tau_{\text {Thermal }} \sim T_{\mathrm{b}} / T_{\mathrm{e}} \sim 0.018$. Given that $\tau_{\text {Thermal }} \propto v^{-2.1}$, we can calculate that $\tau_{\text {Thermal }} \sim 1$ at $v$

\footnotetext{
21 This temperature and the $76 \mathrm{~K}$ derived from Sakamoto et al. (2008) change to $120 \mathrm{~K}$ and $215 \mathrm{~K}$, respectively, if we follow the approach of Downes \& Eckart (2007), which uses the full luminosity and defines the size of the source $\Omega_{\text {source }}$ as $\pi \theta_{\text {source }} / 4 \ln (2)$ with $\theta_{\text {source }}$ the geometric mean between the deconvolved FWHM of the major and minor axis of the west nucleus. That is, the brightness temperature is higher without accounting for inclination effects.
} 
$\sim 5 \mathrm{GHz} .{ }^{22}$ At $18 \mathrm{~cm}, \tau_{\text {Thermal }}>9$, which might help explain the low VLBA flux density from Lonsdale et al. (2006). In addition, as Sakamoto et al. (2008) note, the dust opacity is close to unity at $860 \mu \mathrm{m}$. Thus, the Arp 220 nuclei may be transparent only near the middle of the frequency range $5-350 \mathrm{GHz}$. A case can thus be made that the $33 \mathrm{GHz}$ image presented here is the only existing image that is both optically thin and resolves the nuclei.

\subsection{Evidence at Radio Wavelengths of a Dominant AGN in the Western Nucleus}

In our observations, the western nucleus is more compact with a higher $T_{b}$ than the eastern nucleus. However, consistent with previous VLBI observations, we observe no significant central excess in either Arp 220 images or radial profiles (Figures 1 and 2). Parra et al. (2007) discuss the possibility that one of three VLBI point sources showing a flat spectrum $(\alpha>-0.5)$, could be an AGN. However, that is one of several possibilities that could explain the shape of their spectrum.

Most of the $33 \mathrm{GHz}$ emission that we observe comes from the compact, but still resolved, disks around the nuclei. Specifically, the nuclear beam of the west nucleus contains $20 \%$ of the total flux of the nucleus, while the other $80 \%$ arises from the more extended star-forming regions (Table 1). Our measured $R_{50 d}$ contain similar information (see Table 2). We cannot rule out an AGN in the western nucleus, but if one is present it does not make a dominant, point-like contribution to the overall $33 \mathrm{GHz}$ emission on scales of $\approx 30 \mathrm{pc}$. Similarly, Arp 220 does not exceed the "Eddington" value that might eliminate star formation as a viable power source (see Section 4.3.3). Smith et al. (1998) show that the SN rate and luminosity of Arp 220 are broadly consistent with emission only generated by star formation, though uncertainty in the $\mathrm{IMF}, \mathrm{SN}$ rate, and SFR certainly would still allow an AGN contribution.

No high brightness temperature radio core indicative of an AGN is present. However, given that most AGN are radio-quiet and have weak core emission (e.g., Kellermann et al. 1989; Blundell \& Beasley 1998), the absence of a radio core does not rule out the presence of an AGN. Indeed, several studies at other wavelengths have presented evidence of a possible AGN in Arp 220 (e.g., Iwasawa et al. 2005; Downes \& Eckart 2007; Rangwala et al. 2011; Imanishi \& Saito 2014; Wilson et al. 2014), but to date there is no clear evidence that the putative AGN makes a significant contribution to the bolometric luminosity. If our estimate of $N(\mathrm{H}) \sim 10^{25} \mathrm{~cm}^{-2}$ in Section 4.3 .1 is correct, it would explain why evidence for AGN in Arp 220 has been so elusive. With such high column densities any AGN would be Compton thick and undetectable by standard AGN diagnostic tools.

\section{SUMMARY}

We present new, high-resolution VLA observations of the nearest ULIRG, Arp 220. Our $33 \mathrm{GHz}$ observations measure the light distribution, which originates mostly from synchrotron emission, at a wavelength where optical depth effects are likely negligible. We find exponential profiles with half-light radii of 51 and $35 \mathrm{pc}$ for the eastern and western nucleus, respectively. The distribution of $33 \mathrm{GHz}$ radio emission matches the number density distribution of recent RSNe/SNRs very well. This similarity may result from strong $(\sim \mathrm{mG})$ magnetic

\footnotetext{
$22 \tau_{\text {Thermal }}$ increases to 0.028 and $v$ to $6 \mathrm{GHz}$ if instead $55 \%$ of the $33 \mathrm{GHz}$ emission is thermal (see discussion in Section 4.1).
}

fields, which could yield cooling timescales for CR electrons that are short compared to the diffusion timescale. Adopting the measured $33 \mathrm{GHz}$ sizes as characteristic of the star-forming disks, we derive implied surface densities, $\mathrm{H}$ column densities, and volumetric gas densities that strikingly illustrate the extreme nature of the environment present in Arp 220. Combining our size measurements with unresolved IR measurements, we estimate total fluxes that, although very large, lie well below the conservative predicted values for the Eddingtonlimited "maximal starburst," though this result is sensitive to our assumptions. Regardless, the implied luminosity surface brightness for the west nucleus of Arp 220 is among the most extreme for any measured system. Given the general uncertain evidence to date of a dominant AGN in Arp 220, we conclude that the compact size and disk-like morphology clearly make Arp 220 a prototypical example of the most extreme class of star-forming systems in the local universe.

We thank the anonymous referee for helpful comments that made this paper stronger. We thank Guillermo Damke for his helpful input in the modeling process, Norman Murray for helpful discussions at an early stage of the project, Phil Arras for useful suggestions on Appendix A, and Shane Davis for his input, especially in the development of Appendix B, regarding radiation pressure and the Eddington limit. We thank the NRAO/UVa star formation group (especially Crystal Brogan and Kelsey Johnson) for repeated technical and scientific feedback. We also thank Nick Scoville for detailed discussions of the astrometry of Arp 220. A.S.E., G.C.P., and L.B.-M. were supported by NSF grant AST 1109475. L.B.-M. was also supported by Fulbright and Becas Chile-CONICYT. This research made use of the NASA/IPAC Extragalactic Database (NED), which is operated by the Jet Propulsion Laboratory, California Institute of Technology, under contract with the National Aeronautics and Space Administration, and NASA's Astrophysics Data System Bibliographic Services. The National Radio Astronomy Observatory is a facility of the National Science Foundation operated under cooperative agreement by Associated Universities, Inc.

\section{APPENDIX A}

\section{FLUX THROUGH AN AREA JUST ABOVE AN EXTENDED THIN DISK}

Consider a geometrically thin disk with radius $R$ viewed face on. Then consider a small area parallel to the disk and at distance $d$ above the disk center. The flux, $F$, passing through the area will be

$$
F=\int I \cos (\theta) d \Omega,
$$

where $d \Omega=d \phi \sin \theta d \theta$ is the area subtended by an infinitesimal part of the disk. The factor $\cos \theta$ accounts for the orientation of the area relative to the patch of emitting disk under consideration with $\cos \theta=\left(d / \sqrt{R^{2}+d^{2}}\right)$ just as $\sin \theta=\left(R / \sqrt{R^{2}+d^{2}}\right)$. $I$ is the specific intensity, which for the optically thick case, is just the source function of the disk and is the same for all lines of sight (as the disk fills the beam). In the scenario where radiation pressure is important, we consider that near the disk high optical depth is likely and proceed in the case of Arp $220 .^{23}$

\footnotetext{
23 In the optically thin case, $I$ will depend on the path length through the disk, which is larger by a factor of $\cos \theta$ at high viewing angles. This factor cancels with the directional $\cos \theta$ in Equation (A1) so that the optically thin case yields a different answer.
} 
The integral in Equation (A1) goes from 0 to $2 \pi$ in $\phi$ and 0 to $\sin ^{-1}\left(R / \sqrt{R^{2}+d^{2}}\right)$ in $\theta$. We will immediately change variables so that $x \equiv \sin \theta$ and $d x \equiv \cos \theta d \theta$. Thus,

$$
F=I \int_{0}^{2 \pi} d \phi \int_{0}^{\frac{R}{\sqrt{R^{2}+d^{2}}}} x d x=\pi I \frac{R^{2}}{R^{2}+d^{2}} .
$$

Consider the limit where $d \ll R$, i.e., where the area the flux is passing through lies just above the disk. Then

$$
F_{\text {near }}=\pi I
$$

similar to the well-known relation that the flux at the surface of a black body is $\pi B_{v} \cdot{ }^{24}$ Similarly, at large $d \gg R$, as for an astronomical observation:

$$
F_{\text {far }}=\pi I \frac{R^{2}}{d^{2}},
$$

which is nothing more than the integral of the intensity over the solid angle subtended by the disk. The utility in this calculation is to relate $I$ back to the luminosity, which for an isotropic emitter is just $L=4 \pi d^{2} F_{\text {far }}$ (see the last paragraph of this appendix for some caveats regarding this assumption). Then

$$
F_{\text {far }}=\pi I \frac{R^{2}}{d^{2}}=\frac{L}{4 \pi d^{2}},
$$

so that

$$
I=\frac{L}{4 \pi^{2} R^{2}} \quad \text { and } F_{\text {near }}=\frac{L}{4 \pi R^{2}} .
$$

This $F_{\text {near }}$ is the flux through a surface near the disk and the departure from the perhaps expected $L /\left(2 \pi R^{2}\right)$ is that we have included the $\cos \theta$ term in the original setup to account for the projection of the incident intensity onto the unit area.

When considering real observations cast in terms of the halflight area, $A_{50}$ (and recall that the commonly used size at FWHM for a $2 \mathrm{D}$ Gaussian is $A_{50}$ ) an additional factor enters from the fact that $L_{50}=0.5 L$ for $R_{50}$. Then in general,

$$
F_{\text {near }}=\frac{L}{8 \pi R_{50}^{2}},
$$

is the flux that should be used for considering radiation pressure near a large disk (where large is defined so that $d \ll R$ can hold). In Arp 220, an additional factor of two comes into play if we assume the luminosity is split between the two nuclei with a ratio of $1: 1$. This decreases the relation to $F_{\text {near }}=L /\left(16 \pi R_{50}^{2}\right)$ for this specific case. Note the stark difference, even for the general case, from the commonly adopted $\Sigma_{\mathrm{IR}}=L / \pi R_{50}^{2}$.

In the case of an optically thick disk, the assumption of isotropy is not valid and $L \neq 4 \pi d^{2} F_{\text {far }}$, instead $L=2 \pi d^{2} F_{\text {far }}$. Furthermore, the emission is not isotropically distributed, so that if the disk is inclined by an angle, $i$, with respect to the line of sight, where $i=0$ is a face-on disk, then $L=2 \pi d^{2} F_{\text {far } / \cos }$ $i$. In other words, the emission comes only from the two sides of the disks and an observer finds more flux when the disk is viewed face on (because the constant intensity surface subtends more solid angle). This is a substantial uncertainty for Arp 220 (e.g., see Downes \& Eckart 2007): it appears to host two inclined disks and shows good evidence for optical depth at IR wavelengths. However, we are hesitant to impose

\footnotetext{
$\overline{24} F_{\text {near }}$ will be different by a factor of two in the optically thin case.
}

any correction to the luminosity in the main analysis because we do not know the true geometry of the IR photosphere, which might very plausibly be more spherical and emit more isotropically than the nuclear disks picked out by our $33 \mathrm{GHz}$ observations. Therefore throughout the main text we have used the conventional $L=4 \pi d^{2} F_{\text {far }}$ but we note this substantial uncertainty.

\section{APPENDIX B}

\section{VERTICAL HYDROSTATIC EQUILIBRIUM FOR A SIMPLE RADIATION PRESSURE DOMINATED DISK}

As a simple check on the plausibility of radiation pressure representing the main means of support, we consider vertical hydrostatic equilibrium in a simple gas disk. We consider an infinite slab of surface density $\Sigma$, so that the integrated weight of the column of gas at the midplane is $\pi G \Sigma^{2}$ (here $G$ is the gravitational constant). To a coarse approximation, radiation pressure can counteract this weight with a pressure set by the momentum flux of photons, $F / 2 c$ (where $c$ is the speed of light), multiplied by the total opacity of the gas column, $\kappa \Sigma$, where $\kappa$ is the cross section per unit gas mass. Then,

$$
\pi G \Sigma^{2} \sim \frac{\kappa \Sigma F}{2 c} .
$$

We can then solve for $\kappa$ in terms of the other properties of the disk and the resulting $\kappa$ expresses the required effective opacity in order for radiation pressure to balance the weight of the disk:

$$
\kappa \sim \frac{2 \pi c G \Sigma}{F}
$$

If $F$ and $\Sigma$ are known, comparison of the required $\kappa$ to realistic values represents a zeroth order check of whether radiation pressure represents a viable support mechanism for a system. As a close corollary, if $\kappa$ is known or can be estimated, assessing the degree to which Equation (B1) represents an inequality offers diagnostic of the importance of radiation pressure to the system. Note that $\kappa$, as we have written it, will depend on the dust-to-gas ratio, grain properties, overall opacity, and temperature distribution. In our simplified thin disk geometry, it does not depend directly on the size of the system but all of these properties may vary substantially as a function of disk structure. As a first order approximation, we assume the Semenov et al. (2003) model values serve as a template of typical values that would describe $\kappa$ for the nuclei in Arp 220, but to our knowledge a thorough exploration of $\kappa$ appropriate for the nuclear disks of merging galaxies remains lacking in the literature.

\section{REFERENCES}

\footnotetext{
Alonso-Herrero, A., Rieke, G. H., Rieke, M. J., \& Scoville, N. Z. 2000, ApJ, 532,845

Anantharamaiah, K. R., Viallefond, F., Mohan, N. R., Goss, W. M., \& Zhao, J. H. 2000, ApJ, 537, 613

Armus, L., Charmandaris, V., Bernard-Salas, J., et al. 2007, ApJ, 656, 148 Blundell, K. M., \& Beasley, A. J. 1998, MNRAS, 299, 165

Clemens, M. S., Scaife, A., Vega, O., \& Bressan, A. 2010, MNRAS, 405, 887 Clements, D. L., McDowell, J. C., Shaked, S., et al. 2002, ApJ, 581, 974 Condon, J. J. 1992, ARA\&A, 30, 575

Condon, J. J., Huang, Z.-P., Yin, Q. F., \& Thuan, T. X. 1991, ApJ, 378, 65 Davis, S. W., Jiang, Y.-F., Stone, J. M., \& Murray, N. 2014, ApJ, 796, 107 Díaz-Santos, T., Armus, L., Charmandaris, V., et al. 2013, ApJ, 774, 68 Downes, D., \& Eckart, A. 2007, A\&A, 468, 57

Downes, D., \& Solomon, P. M. 1998, ApJ, 507, 615

Engel, H., Davies, R. I., Genzel, R., et al. 2011, ApJ, 729, 58
} 
Genzel, R., Tacconi, L. J., Rigopoulou, D., Lutz, D., \& Tecza, M. 2001, ApJ, 563,527

González-Alfonso, E., Fischer, J., Graciá-Carpio, J., et al. 2012, A\&A, 541, A4

Haas, M., Klaas, U., Müller, S. A. H., Chini, R., \& Coulson, I. 2001, A\&A, 367, L9

Herrero-Illana, R., Pérez-Torres, M. Á., \& Alberdi, A. 2012, A\&A, 540, L15

Hopkins, P. F., Murray, N., Quataert, E., \& Thompson, T. A. 2010, MNRAS, 401, L19

Ilovaisky, S. A., \& Lequeux, J. 1972, A\&A, 20, 347

Imanishi, M., \& Saito, Y. 2014, ApJ, 780, 106

Iwasawa, K., Sanders, D. B., Evans, A. S., et al. 2005, MNRAS, 357, 565

Kellermann, K. I., Sramek, R., Schmidt, M., Shaffer, D. B., \& Green, R. 1989, AJ, 98, 1195

Krumholz, M. R., \& Thompson, T. A. 2013, MNRAS, 434, 2329

Leroy, A. K., Evans, A. S., Momjian, E., et al. 2011, ApJL, 739, L25

Lonsdale, C. J., Diamond, P. J., Thrall, H., et al. 2006, ApJ, 647, 185

Mazzarella, J. M., Soifer, B. T., Graham, J. R., et al. 1992, AJ, 103, 413

McBride, J., Quataert, E., Heiles, C., \& Bauermeister, A. 2014, ApJ, 780, 182

McMullin, J. P., Waters, B., Schiebel, D., Young, W., \& Golap, K. 2007, in ASP

Conf. Ser. 376, Astronomical Data Analysis Software and Systems XVI, ed.

R. A. Shaw, F. Hill, \& D. J. Bell (San Francisco, CA: ASP), 127

Meurer, G. R., Heckman, T. M., Lehnert, M. D., Leitherer, C., \& Lowenthal, J. 1997, AJ, 114, 54

Mundell, C. G., Ferruit, P., \& Pedlar, A. 2001, ApJ, 560, 168

Murphy, E. J. 2009, ApJ, 706, 482

Murphy, E. J. 2013, ApJ, 777, 58

Murphy, E. J., Bremseth, J., Mason, B. S., et al. 2012, ApJ, 761, 97

Murphy, E. J., Helou, G., Braun, R., et al. 2006, ApJL, 651, L111
Norris, R. P. 1988, MNRAS, 230, 345

Papadopoulos, P. P., van der Werf, P., Xilouris, E., Isaak, K. G., \& Gao, Y. 2012, ApJ, 751, 10

Parra, R., Conway, J. E., Diamond, P. J., et al. 2007, ApJ, 659, 314

Pooley, G. G. 1969, MNRAS, 144, 101

Rangwala, N., Maloney, P. R., Glenn, J., et al. 2011, ApJ, 743, 94

Robishaw, T., Quataert, E., \& Heiles, C. 2008, ApJ, 680, 981

Rodríguez-Rico, C. A., Goss, W. M., Viallefond, F., et al. 2005, ApJ, 633, 198

Sakamoto, K., Scoville, N. Z., Yun, M. S., et al. 1999, ApJ, 514, 68

Sakamoto, K., Wang, J., Wiedner, M. C., et al. 2008, ApJ, 684, 957

Sanders, D. B., Mazzarella, J. M., Kim, D.-C., et al. 2003, AJ, 126, 1607

Sanders, D. B., \& Mirabel, I. F. 1996, ARA\&A, 34, 749

Sault, R. J., \& Wieringa, M. H. 1994, A\&AS, 108, 585

Scoville, N. 2003, JKAS, 36, 167

Scoville, N. Z., Evans, A. S., Dinshaw, N., et al. 1998, ApJL, 492, L107

Semenov, D., Henning, T., Helling, C., Ilgner, M., \& Sedlmayr, E. 2003, A\&A, 410, 611

Smith, H. E., Lonsdale, C. J., Lonsdale, C. J., \& Diamond, P. J. 1998, ApJL, 493, L17

Socrates, A., Davis, S. W., \& Ramirez-Ruiz, E. 2008, ApJ, 687, 202

Socrates, A., \& Sironi, L. 2013, ApJL, 772, L21

Soifer, B. T., Neugebauer, G., Matthews, K., et al. 1999, ApJ, 513, 207

Soifer, B. T., Neugebauer, G., Matthews, K., et al. 2000, AJ, 119, 509

Spoon, H. W. W., Marshall, J. A., Houck, J. R., et al. 2007, ApJL, 654, L49

Thompson, T. A., Quataert, E., \& Murray, N. 2005, ApJ, 630, 167

Williams, P. K. G., \& Bower, G. C. 2010, ApJ, 710, 1462

Wilson, C. D., Rangwala, N., Glenn, J., et al. 2014, ApJL, 789, L36 